\title{
Gerstenhaber algebra structure on the Hochschild cohomology of quadratic string algebras
}

\author{
María Julia Redondo and Lucrecia Román *†
}

\begin{abstract}
We describe the Gerstenhaber algebra structure on the Hochschild cohomology $\operatorname{HH}^{*}(A)$ when $A$ is a quadratic string algebra. First we compute the Hochschild cohomology groups using Barzdell's resolution and we describe generators of these groups. Then we construct comparison morphisms between the bar resolution and Bardzell's resolution in order to get formulae for the cup product and the Lie bracket. We find conditions on the bound quiver associated to string algebras in order to get non-trivial structures.
\end{abstract}

2010 MSC: 16E40; 16W99

\section{Introduction}

Let $A$ be an associative, finite dimensional algebra over an algebraically closed field $k$. By a fundamental result in representation theory it is well known that there exists a finite quiver $Q$ such that $A$ is Morita equivalent to $k Q / I$, where $k Q$ is the path algebra of $Q$ and $I$ is an admissible two-sided ideal of $k Q$, see for instance [2, Theorem 3.7]. The pair $(Q, I)$ is called a bound quiver of $A$.

A finite dimensional algebra is called biserial if the radical of every projective indecomposable module is the sum of two uniserial modules whose intersection is simple or zero, see [14]. These algebras have been studied by several authors and from different points of view since there are a lot of natural examples of algebras which turn out to be of this kind: Nakayama algebras [23, Kawada algebras [19, 27], blocks of group algebras with cyclic or dihedral defect groups [18, 13, 26], iterated tilted algebras of type $A$ and $\tilde{A}[1,3$, Brauer graph algebras [31] and algebras appearing in the Gel'fand-Ponomarev classification of Harish-Chandra modules over the Lorentz group [15].

\footnotetext{
*Instituto de Matemática (INMABB), Departamento de Matemática, Universidad Nacional del Sur (UNS)CONICET, Bahía Blanca, Argentina.E-mail address: mredondo@criba.edu.ar,lroman@uns.edu.ar

${ }^{\dagger}$ The first author is a researcher and the second author has a fellowship from CONICET, Argentina. This work has been supported by the project PICT-2011-1510.
} 
The representation theory of these algebras was first studied by Gel'fand and Ponomarev in [15]: they have provided the methods in order to classify all their indecomposable representations. This classification shows that biserial algebras are always tame, see also [12]. They are an important class of algebras whose representation theory has been very well described, see [3, 8].

The subclass of special biserial algebras was studied by Skowroński and Waschbüsch in [34] where they characterize the biserial algebras of finite representation type. The definition of these algebras can be given in terms of conditions on the bound quiver $(Q, I)$ associated (see Section 2.2). A classification of the special biserial algebras which are minimal representation-infinite has been given by Ringel in [28. This class of algebras has played an important rôle in the study of self-injective algebras, and their representation theory is well understood. There is a beautiful description of all finite-dimensional indecomposable modules over special biserial algebras: they are either string modules or band modules or non-uniserial projective-injective modules, see [8, 38].

Since $A$ is an algebra over a field $k$, the Hochschild cohomology groups $\operatorname{HH}^{n}(A, M)$ with coefficients in an $A$-bimodule $M$ can be identified with the groups $\operatorname{Ext}_{A-A}^{n}(A, M)$. In particular, if $M$ is the $A$-bimodule $A$, we simply write $\mathrm{HH}^{n}(A)$. Even though the computation of the Hochschild cohomology groups $\mathrm{HH}^{n}(A)$ is rather complicated, some approaches have been successful when the algebra $A$ is given by a quiver with relations. For instance, explicit formula for the dimensions of $\mathrm{HH}^{n}(A)$ in terms of those combinatorial data have been found in [6, 9, 10, 11, 17, 24]. In particular, Hochschild cohomology of special biserial algebras has been considered in [7, 20, 35].

In the particular case of monomial algebras, that is, algebras $A=k Q / I$ where $I$ can be chosen as generated by paths, one has a detailed description of a minimal resolution of the $A$-bimodule $A$ : Bardzell's resolution [5].

An algebra is called a string algebra if it is Morita equivalent to a monomial special biserial algebra $k Q / I$, and it is called quadratic if the ideal $I$ is generated by paths of length two. In general, the computation of the Hochschild cohomology groups using Bardzell's resolution may lead to hard combinatorial computations. However, for quadratic string algebras the resolution, and the complex associated, are easier to handle.

The sum $\mathrm{HH}^{*}(A)=\bigoplus_{n \geq 0} \mathrm{HH}^{n}(A)$ is a Gerstenhaber algebra, that is, it is a graded commutative ring via the cup product, a graded Lie algebra via the bracket, and these two structures are related, see [16. So far there are only a few classes of algebras in the literature where the Gerstenhaber algebra structure on Hochschild cohomology has been determined explicitly, see [7, 21, 22, 30, 35, 36, 32, 37, 39]. In [25] we compute the Hochschild cohomology groups of a triangular string algebra, and we prove that its ring structure is trivial.

The purpose of this paper is to study the Gerstenhaber algebra structure on the Hochschild cohomology of a quadratic string algebra $A$, that is, we describe explicitly the ring and the Lie algebra structure of the Hochschild cohomology of $A$, and we delete the triangular condition in order to get non-trivial structures. The main results show the dimension of the 
Hochschild cohomology groups $\mathrm{HH}^{n}(A)$, see Theorems 3.13 and 3.14. These computations are done using Bardzell's resolution, and allow us to give an explicit basis for each cohomology group. Furthermore, we construct a comparison morphism between the bar resolution and Bardzell's resolution which lead us to the formulae for the cup product and the Lie bracket.

Finally we describe explicit non-zero elements in the Hochschild cohomology whose cup product and Lie bracket are non-zero, that is, we define $\mathcal{G}_{n}$ to be the set of gentle pairs (see Definition $3.8(\mathrm{a})$ ) and we prove the following theorems.

Theorem (Theorem 4.8) Let $A=k Q / I$ be a quadratic string algebra and $\mathcal{G}_{n} \neq \emptyset$ for some $n>0$. Then the cup product defined in $\mathrm{HH}^{*}(A)$ is non-trivial. More precisely,

(i) if $n$ is even and char $k \neq 2, \mathrm{HH}^{s_{1} n}(A) \cup \mathrm{HH}^{s_{2} n}(A) \neq 0$;

(ii) if $n$ is odd and char $k \neq 2, \mathrm{HH}^{2 s_{1} n}(A) \cup \mathrm{HH}^{2 s_{2} n}(A) \neq 0$;

(iii) if $\operatorname{char} k=2, \mathrm{HH}^{s_{1} n}(A) \cup \mathrm{HH}^{s_{2} n}(A) \neq 0$

for any $s_{1}, s_{2} \geq 1$.

Theorem (Theorem 4.9) Let char $k=0$ and let $A=k Q / I$ be a quadratic string algebra such that $\mathcal{G}_{n} \neq \emptyset$ for some $n>0$. Then the Lie bracket defined in $\mathrm{HH}^{*}(A)$ is non-trivial. More precisely,

(i) if $n$ is even, $\left[\mathrm{HH}^{s_{1} n+1}(A), \mathrm{HH}^{s_{2} n+1}(A)\right] \neq 0$;

(ii) if $n$ is odd, $\left[\mathrm{HH}^{2 s_{1} n+1}(A), \mathrm{HH}^{2 s_{2} n+1}(A)\right] \neq 0$

for any $s_{1}, s_{2} \geq 1, s_{1} \neq s_{2}$.

These proofs are given in terms of representatives $u_{s n}$ and $u_{s n+1}$ of non-zero elements in $\mathrm{HH}^{s n}(A)$ and $\mathrm{HH}^{s n+1}(A)$ respectively such that

$$
u_{s_{1} n} \cup u_{s_{2} n}=u_{\left(s_{1}+s_{2}\right) n} \quad \text { and } \quad\left[u_{s_{1} n+1}, u_{s_{2} n+1}\right]=\lambda u_{\left(s_{1}+s_{2}\right) n+1}
$$

for some scalar $\lambda \in k$.

The paper is organized as follows. In Section 2 we introduce all the necessary terminology. In Section 3 we recall the resolution given by Bardzell for monomial algebras in [5] and we present all the computations that lead us to Theorems 3.13 and 3.14 where we present the dimension of all the Hochschild cohomology groups of quadratic string algebras. In Section 4 we describe the ring and the Lie structure of the Hochschild cohomology of these algebras and we find conditions on the bound quiver associated in order to get non-trivial structures. 


\section{Preliminaries}

\subsection{Quivers and relations}

Let $Q$ be a connected finite quiver with a set of vertices $Q_{0}$, a set of arrows $Q_{1}$ and $s, t$ : $Q_{1} \rightarrow Q_{0}$ be the maps associating to each arrow $\alpha$ its source $s(\alpha)$ and its target $t(\alpha)$. A path $w$ of length $l$ is a sequence of $l$ arrows $\alpha_{1} \ldots \alpha_{l}$ such that $t\left(\alpha_{i}\right)=s\left(\alpha_{i+1}\right)$. We denote by $|w|$ the length of the path $w$. We put $s(w)=s\left(\alpha_{1}\right)$ and $t(w)=t\left(\alpha_{l}\right)$. For any vertex $x$ we consider $e_{x}$ the trivial path of length zero and we put $s\left(e_{x}\right)=t\left(e_{x}\right)=x$. An oriented cycle is a non-trivial path $w$ such that $s(w)=t(w)$.

The path algebra $k Q$ is the $k$-vector space with basis the set of paths in $Q$; the product on the basis elements is given by the concatenation of the sequences of arrows of the paths $w$ and $w^{\prime}$ if they form a path (namely, if $t(w)=s\left(w^{\prime}\right)$ ) and zero otherwise. Vertices form a complete set of orthogonal idempotents of $k Q$. Let $F$ be the two-sided ideal of $k Q$ generated by the arrows of $Q$. A two-sided ideal $I$ of $k Q$ is said to be admissible if there exists an integer $m \geq 2$ such that $F^{m} \subseteq I \subseteq F^{2}$. The elements in $I$ are called relations, $k Q / I$ is called a monomial algebra if the ideal $I$ is generated by paths, and a relation is called quadratic if it is a path of length two.

By a fundamental result in representation theory it is well known that if $A$ is a basic, indecomposable, finite dimensional algebra over an algebraically closed field $k$, then there exists a unique finite connected quiver $Q$ and a surjective morphism of $k$-algebras $\nu: k Q \rightarrow A$, which is not unique in general, with $I_{\nu}=\operatorname{Ker} \nu$ admissible, see for instance [2, Theorem 3.7]. The pair $\left(Q, I_{\nu}\right)$ is called a presentation of $A$.

\section{$2.2 \quad$ String algebras}

Recall from [34] that a bound quiver $(Q, I)$ is special biserial if it satisfies the following conditions:

S1) Each vertex in $Q$ is the source of at most two arrows and the target of at most two arrows;

S2) For an arrow $\alpha$ in $Q$ there is at most one arrow $\beta$ and at most one arrow $\gamma$ such that $\alpha \beta \notin I$ and $\gamma \alpha \notin I$.

If the ideal $I$ is generated by paths, the bound quiver $(Q, I)$ is string.

An algebra is called special biserial (or string) if it is Morita equivalent to a path algebra $k Q / I$ with $(Q, I)$ a special biserial bound quiver (or a string bound quiver, respectively).

Since Hochschild cohomology is invariant under Morita equivalence, whenever we deal with a string algebra $A$ we will assume that it is given by a string presentation $A=k Q / I$ with $(Q, I)$ satisfying the previous conditions. 
In this paper we are interested in quadratic string algebras, that is, the ideal $I$ is generated by paths of length two. We fix a minimal set $\mathcal{R}$ of paths that generates the ideal $I$. Moreover, we denote by $\mathcal{P}$ the set of paths in $Q$ such that the set $\{\gamma+I, \gamma \in \mathcal{P}\}$ is a basis of $A=k Q / I$. It is clear that $Q_{0} \cup Q_{1} \subseteq \mathcal{P}$ since $I \subseteq F^{2}$.

\section{Hochschild cohomology groups}

\subsection{Bardzell's resolution for quadratic string algebras}

We recall that the Hochschild cohomology groups $\mathrm{HH}^{n}(A)$ of an algebra $A$ are the groups $\operatorname{Ext}_{A-A}^{n}(A, A), n \geq 0$. Since string algebras are monomial algebras, their Hochschild cohomology groups can be computed using a convenient minimal projective resolution of $A$ as $A$-bimodule constructed by Bardzell in [5, Theorem 4.1]. In the particular case of quadratic string algebras, this minimal resolution is the following:

$$
\cdots \longrightarrow A \otimes k A P_{n} \otimes A \stackrel{d_{n}}{\longrightarrow} A \otimes k A P_{n-1} \otimes A \longrightarrow \cdots \longrightarrow A \otimes k A P_{0} \otimes A \stackrel{\mu}{\longrightarrow} A \longrightarrow 0
$$

where $k A P_{0}=k Q_{0}, k A P_{1}=k Q_{1}$, for $n \geq 2, k A P_{n}$ is the vector space generated by the set

$$
A P_{n}=\left\{\alpha_{1} \alpha_{2} \cdots \alpha_{n}: \alpha_{i} \alpha_{i+1} \in I, 1 \leq i<n\right\},
$$

all tensor products are taken over $E=k Q_{0}$, the subalgebra of $A$ generated by the vertices, and the $A$-bimodule morphisms are

$$
\begin{aligned}
\mu\left(1 \otimes e_{i} \otimes 1\right) & =e_{i}, \\
d_{1}(1 \otimes \alpha \otimes 1) & =\alpha \otimes e_{t(\alpha)} \otimes 1-1 \otimes e_{s(\alpha)} \otimes \alpha \\
d_{n}\left(1 \otimes \alpha_{1} \cdots \alpha_{n} \otimes 1\right) & =\alpha_{1} \otimes \alpha_{2} \cdots \alpha_{n} \otimes 1+(-1)^{n} 1 \otimes \alpha_{1} \cdots \alpha_{n-1} \otimes \alpha_{n} .
\end{aligned}
$$

The $E$ - $A$-bilinear map $c: A \otimes k A P_{n-1} \otimes A \rightarrow A \otimes k A P_{n} \otimes A$ defined by

$$
c\left(a \otimes \alpha_{1} \cdots \alpha_{n-1} \otimes 1\right)= \begin{cases}b \otimes \alpha_{0} \alpha_{1} \cdots \alpha_{n-1} \otimes 1 & \text { if } a=b \alpha_{0} \text { and } \alpha_{0} \alpha_{1} \in I, \\ 0 & \text { otherwise }\end{cases}
$$

is a contracting homotopy, see [33, Theorem 1] for more details.

The Hochschild complex, obtained by applying $\operatorname{Hom}_{A-A}(-, A)$ to the resolution we have just described and using the isomorphisms

$$
\operatorname{Hom}_{A-A}\left(A \otimes k A P_{n} \otimes A, A\right) \simeq \operatorname{Hom}_{E-E}\left(k A P_{n}, A\right)
$$

is

$$
0 \longrightarrow \operatorname{Hom}_{E-E}\left(k A P_{0}, A\right) \stackrel{F_{1}}{\longrightarrow} \operatorname{Hom}_{E-E}\left(k A P_{1}, A\right) \stackrel{F_{2}}{\longrightarrow} \operatorname{Hom}_{E-E}\left(k A P_{2}, A\right) \rightarrow \cdots
$$


where

$$
\begin{aligned}
F_{1}(f)(\alpha) & =\alpha f\left(e_{t(\alpha)}\right)-f\left(e_{s(\alpha)}\right) \alpha \\
F_{n}(f)\left(\alpha_{1} \cdots \alpha_{n}\right) & =\alpha_{1} f\left(\alpha_{2} \cdots \alpha_{n}\right)+(-1)^{n} f\left(\alpha_{1} \cdots \alpha_{n-1}\right) \alpha_{n} .
\end{aligned}
$$

\subsection{Computations}

In order to describe the ring and the Lie algebra structure of the Hochschild cohomology $\mathrm{HH}^{*}(A)$ of a quadratic string algebra, we need a convenient description of the previous complex. In this section we will describe explicit basis of these $k$-vector spaces and study the behavior of the maps between them in order to get information about kernels and images.

For any pair $X, Y$ of sets of paths in $Q$ we denote by $(X / / Y)$ the set of pairs $(\rho, \gamma) \in X \times Y$ such that $\rho, \gamma$ are parallel paths in $Q$, that is

$$
(X / / Y)=\{(\rho, \gamma) \in X \times Y: s(\rho)=s(\gamma), t(\rho)=t(\gamma)\}
$$

Recall that we have fixed a set $\mathcal{P}$ of paths in $Q$ such that the set $\{\gamma+I: \gamma \in \mathcal{P}\}$ is a basis of $A=k Q / I$. For any $m \geq 0$ we denote by $\mathcal{P}_{m}$ the set of paths in $\mathcal{P}$ whose length is greater than or equal to $m$. Then

$$
\left(X / / \mathcal{P}_{m}\right)=\{(\rho, \gamma) \in X \times \mathcal{P}: s(\rho)=s(\gamma), t(\rho)=t(\gamma),|\gamma| \geq m\}
$$

and hence $(X / / \mathcal{P})=\left(X / / Q_{0}\right) \sqcup\left(X / / \mathcal{P}_{1}\right)=\left(X / / Q_{0}\right) \sqcup\left(X / / Q_{1}\right) \sqcup\left(X / / \mathcal{P}_{2}\right)$, where $\sqcup$ depicts disjoint union.

Example 3.1. Let $A=k Q / I$ where $Q$ is the quiver

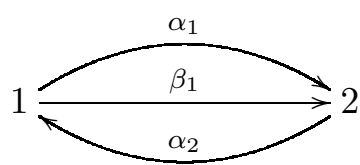

and $I=<\alpha_{1} \alpha_{2}, \alpha_{2} \alpha_{1}, \beta_{1} \alpha_{2}>$. The sets $\left(A P_{n} / / \mathcal{P}\right)$ are

$$
\begin{aligned}
& \left(A P_{0} / / Q_{0}\right)=\left\{\left(e_{1}, e_{1}\right),\left(e_{2}, e_{2}\right)\right\}, \\
& \left(A P_{0} / / \mathcal{P}_{1}\right)=\left\{\left(e_{2}, \alpha_{2} \beta_{1}\right)\right\}
\end{aligned}
$$

and, for $i \geq 0$,

$$
\begin{aligned}
\left(A P_{2 i} / / Q_{0}\right)= & \left\{\left(\left(\alpha_{1} \alpha_{2}\right)^{i}, e_{1}\right),\left(\left(\alpha_{2} \alpha_{1}\right)^{i}, e_{2}\right),\left(\beta_{1} \alpha_{2}\left(\alpha_{1} \alpha_{2}\right)^{i-1}, e_{1}\right)\right\}, \\
\left(A P_{2 i} / / \mathcal{P}_{1}\right)= & \left\{\left(\left(\alpha_{2} \alpha_{1}\right)^{i}, \alpha_{2} \beta_{1}\right)\right\}, \\
\left(A P_{2 i+1} / / Q_{0}\right)= & \emptyset \\
\left(A P_{2 i+1} / / \mathcal{P}_{1}\right)= & \left\{\left(\left(\alpha_{1} \alpha_{2}\right)^{i} \alpha_{1}, \alpha_{1}\right),\left(\left(\alpha_{1} \alpha_{2}\right)^{i} \alpha_{1}, \beta_{1}\right),\left(\left(\alpha_{2} \alpha_{1}\right)^{i} \alpha_{2}, \alpha_{2}\right),\right. \\
& \left.\left(\beta_{1}\left(\alpha_{2} \alpha_{1}\right)^{i}, \alpha_{1}\right),\left(\beta_{1}\left(\alpha_{2} \alpha_{1}\right)^{i}, \beta_{1}\right)\right\} .
\end{aligned}
$$


Example 3.2. Let $A=k Q / I$ where $Q$ is the quiver

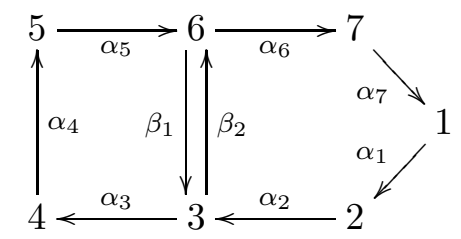

and $I=<\alpha_{i} \alpha_{i+1}>_{\{i=1, \cdots, 6\}}+<\beta_{1} \beta_{2}, \beta_{2} \beta_{1}>$. The sets $\left(A P_{n} / / \mathcal{P}\right)$ are

$$
\begin{aligned}
\left(A P_{0} / / Q_{0}\right) & =\left\{\left(e_{i}, e_{i}\right)\right\}_{\{i=1, \cdots, 7\}}, \\
\left(A P_{0} / / \mathcal{P}_{1}\right) & =\emptyset \\
\left(A P_{1} / / Q_{0}\right) & =\emptyset \\
\left(A P_{1} / / \mathcal{P}_{1}\right) & =\left\{\left(\alpha_{i}, \alpha_{i}\right)\right\}_{\{i=1, \cdots, 7\}} \sqcup\left\{\left(\beta_{i}, \beta_{i}\right)\right\}_{\{i=1,2\}}, \\
\left(A P_{2 i} / / Q_{0}\right) & =\left\{\left(\left(\beta_{1} \beta_{2}\right)^{i}, e_{6}\right),\left(\left(\beta_{2} \beta_{1}\right)^{i}, e_{3}\right)\right\} \quad \text { for } i \geq 1, \\
\left(A P_{4} / / \mathcal{P}_{1}\right) & =\left\{\left(\alpha_{2} \alpha_{3} \alpha_{4} \alpha_{5}, \alpha_{2} \beta_{2}\right),\left(\alpha_{3} \alpha_{4} \alpha_{5} \alpha_{6}, \beta_{2} \alpha_{6}\right)\right\} \\
\left(A P_{2 i} / / \mathcal{P}_{1}\right) & =\emptyset \quad \text { for } i \geq 1, i \neq 2, \\
\left(A P_{7} / / Q_{0}\right) & =\left\{\left(\alpha_{1} \alpha_{2} \ldots \alpha_{6} \alpha_{7}, e_{1}\right)\right\} \\
\left(A P_{2 i+1} / / Q_{0}\right) & =\emptyset \quad \text { for } i \geq 1, i \neq 3, \\
\left(A P_{3} / / \mathcal{P}_{1}\right) & =\left\{\left(\alpha_{3} \alpha_{4} \alpha_{5}, \beta_{2}\right),\left(\beta_{1} \beta_{2} \beta_{1}, \beta_{1}\right),\left(\beta_{2} \beta_{1} \beta_{2}, \beta_{2}\right)\right\} \\
\left(A P_{5} / / \mathcal{P}_{1}\right) & =\left\{\left(\alpha_{2} \alpha_{3} \alpha_{4} \alpha_{5} \alpha_{6}, \alpha_{2} \beta_{2} \alpha_{6}\right),\left(\left(\beta_{1} \beta_{2}\right)^{2} \beta_{1}, \beta_{1}\right),\left(\left(\beta_{2} \beta_{1}\right)^{2} \beta_{2}, \beta_{2}\right)\right\}, \\
\left(A P_{2 i+1} / / \mathcal{P}_{1}\right) & =\left\{\left(\left(\beta_{1} \beta_{2}\right)^{i} \beta_{1}, \beta_{1}\right),\left(\left(\beta_{2} \beta_{1}\right)^{i} \beta_{2}, \beta_{2}\right)\right\} \quad \text { for } i>2 .
\end{aligned}
$$

Observe that the $k$-vector spaces $\operatorname{Hom}_{E-E}\left(k A P_{n}, A\right)$ and $k\left(A P_{n} / / \mathcal{P}\right)$ are isomorphic: the basis element $(\rho, \gamma) \in\left(A P_{n} / / \mathcal{P}\right)$ corresponds to the morphism $f_{(\rho, \gamma)}$ in $\operatorname{Hom}_{E-E}\left(k A P_{n}, A\right)$ defined by

$$
f_{(\rho, \gamma)}(w)= \begin{cases}\gamma & \text { if } w=\rho \\ 0 & \text { otherwise }\end{cases}
$$

Taking into account these isomorphisms, for any $n \geq 0$ we can construct commutative diagrams

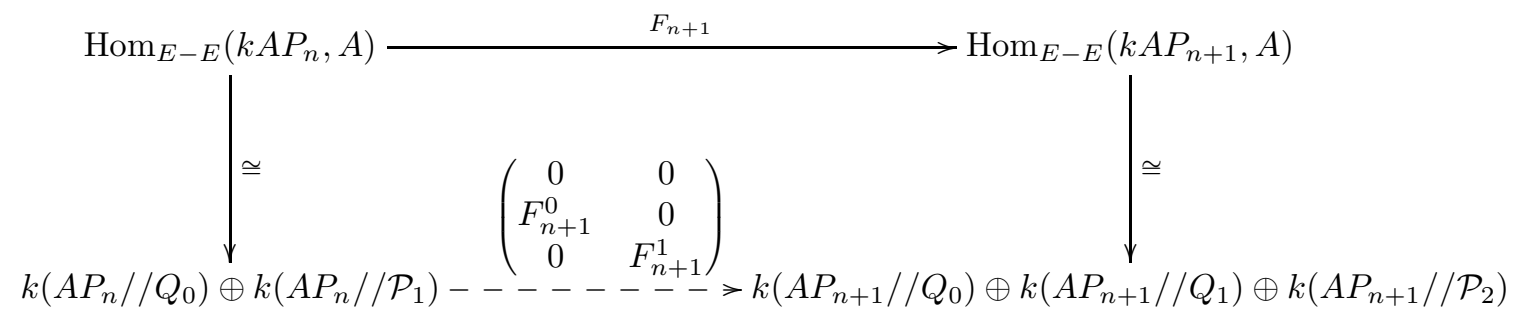


where

$$
\begin{gathered}
F_{1}^{0}\left(e_{r}, e_{r}\right)=\sum_{\left\{\beta \in Q_{1}: t(\beta)=r\right\}}(\beta, \beta)-\sum_{\left\{\beta \in Q_{1}: s(\beta)=r\right\}}(\beta, \beta), \\
F_{1}^{1}\left(e_{r}, \gamma\right)=\sum_{\left\{\beta \in Q_{1}: t(\beta)=r\right\}}(\beta, \beta \gamma)-\sum_{\left\{\beta \in Q_{1}: s(\beta)=r\right\}}(\beta, \gamma \beta), \\
F_{n+1}^{0}\left(\alpha_{1} \cdots \alpha_{n}, e_{s\left(\alpha_{1}\right)}\right) \\
=\sum_{\substack{\left\{\beta \in Q_{1}: \beta \alpha_{1} \in I, t(\beta)=s\left(\alpha_{1}\right)\right\}}}\left(\beta \alpha_{1} \cdots \alpha_{n}, \beta\right)+(-1)^{n+1} \sum_{\substack{\left\{\beta \in Q_{1}: \alpha_{n} \beta \in I, s(\beta)=s\left(\alpha_{1}\right)\right\}}}\left(\alpha_{1} \cdots \alpha_{n} \beta, \beta\right), \\
F_{n+1}^{1}\left(\alpha_{1} \cdots \alpha_{n}, \gamma\right) \\
=\sum_{\substack{\left\{\beta \in Q_{1}: \\
\beta \alpha_{1} \in I, \beta \gamma \notin I\right\}}}\left(\beta \alpha_{1} \cdots \alpha_{n}, \beta \gamma\right)+(-1)^{n+1} \sum_{\substack{\left\{\beta \in Q_{1}: \\
\alpha_{n} \beta \in I, \gamma \beta \notin I\right\}}}\left(\alpha_{1} \cdots \alpha_{n} \beta, \gamma \beta\right) .
\end{gathered}
$$

Now we will introduce several subsets of $\left(A P_{n} / / \mathcal{P}\right)$ in order to get a nice description of the kernel and the image of $F_{n+1}^{0}$ and $F_{n+1}^{1}$.

For $n=0$ we have that $\left(A P_{0} / / \mathcal{P}\right)=\left(Q_{0} / / \mathcal{P}\right)$. Since $A$ is finite dimensional and quadratic, if $\left(e_{s\left(\alpha_{1}\right)}, \alpha_{1} \cdots \alpha_{m}\right) \in\left(Q_{0} / / \mathcal{P}_{1}\right)$ then $\alpha_{m} \alpha_{1} \in I$.

For $n=1,\left(A P_{1} / / \mathcal{P}\right)=\left(Q_{1} / / \mathcal{P}\right)$, and we consider the following partition

$$
\left(Q_{1} / / \mathcal{P}\right)=(1,1)_{1} \sqcup(0,0)_{1} \sqcup(1,0)_{1} \sqcup(0,1)_{1}
$$

defined as follows: if $(\alpha, \gamma) \in\left(Q_{1} / / \mathcal{P}\right)$, we wonder if the path $\gamma$ starts or ends with the arrow $\alpha$, that is,

$$
\begin{aligned}
& (1,1)_{1}=\left\{(\alpha, \alpha): \alpha \in Q_{1}\right\}, \\
& (0,0)_{1}=\left\{(\alpha, \gamma) \in\left(Q_{1} / / \mathcal{P}\right): \gamma \notin \alpha k Q \cup k Q \alpha\right\}, \\
& (1,0)_{1}=\left\{(\alpha, \gamma) \in\left(Q_{1} / / \mathcal{P}\right): \gamma \in \alpha k Q, \gamma \notin k Q \alpha\right\}, \\
& (0,1)_{1}=\left\{(\alpha, \gamma) \in\left(Q_{1} / / \mathcal{P}\right): \gamma \notin \alpha k Q, \gamma \in k Q \alpha\right\} .
\end{aligned}
$$

For any $n \geq 2$, if $(\rho, \gamma) \in\left(A P_{n} / / \mathcal{P}\right)$ then $\rho$ is a path of length greater than or equal to 2 , that is, $\rho=\alpha_{1} \hat{\rho} \alpha_{2}$ and wondering if the path $\gamma$ starts with the arrow $\alpha_{1}$ or ends with the arrow $\alpha_{2}$, we get the following partition

$$
\left(A P_{n} / / \mathcal{P}\right)=(0,0)_{n} \sqcup(1,0)_{n} \sqcup(0,1)_{n} \sqcup(1,1)_{n}
$$


where

$$
\begin{aligned}
& (0,0)_{n}=\left\{(\rho, \gamma) \in\left(A P_{n} / / \mathcal{P}\right): \rho=\alpha_{1} \hat{\rho} \alpha_{2} \text { and } \gamma \notin \alpha_{1} k Q \cup k Q \alpha_{2}\right\}, \\
& (1,0)_{n}=\left\{(\rho, \gamma) \in\left(A P_{n} / / \mathcal{P}\right): \rho=\alpha_{1} \hat{\rho} \alpha_{2} \text { and } \gamma \in \alpha_{1} k Q, \gamma \notin k Q \alpha_{2}\right\}, \\
& (0,1)_{n}=\left\{(\rho, \gamma) \in\left(A P_{n} / / \mathcal{P}\right): \rho=\alpha_{1} \hat{\rho} \alpha_{2} \text { and } \gamma \notin \alpha_{1} k Q, \gamma \in k Q \alpha_{2}\right\}, \\
& (1,1)_{n}=\left\{(\rho, \gamma) \in\left(A P_{n} / / \mathcal{P}\right): \rho=\alpha_{1} \hat{\rho} \alpha_{2} \text { and } \gamma \in \alpha_{1} k Q \cap k Q \alpha_{2}\right\} .
\end{aligned}
$$

We also have to distinguish elements inside each of the previous sets taking into account the following definitions:

$$
\begin{aligned}
{ }^{+}(X / / \mathcal{P}) & =\left\{(\rho, \gamma) \in(X / / \mathcal{P}): Q_{1} \gamma \not \subset I\right\}, \\
{ }^{-}(X / / \mathcal{P}) & =\left\{(\rho, \gamma) \in(X / / \mathcal{P}): Q_{1} \gamma \subset I\right\} .
\end{aligned}
$$

In an analogous way we define $(X / / \mathcal{P})^{+},(X / / \mathcal{P})^{-},{ }^{+}(X / / \mathcal{P})^{+}={ }^{+}(X / / \mathcal{P}) \cap(X / / \mathcal{P})^{+}$and so on.

Example 3.3. For the algebra presented in Example 3.1 we have that, for $n>0$,

$$
\begin{aligned}
& +(0,0)_{n}^{+}= \begin{cases}\left(A P_{n} / / Q_{0}\right) & \text { if } n \text { is even } \\
\emptyset & \text { otherwise }\end{cases} \\
& +(0,0)_{n}^{-}=\left\{\begin{array}{lc}
\left\{\left(\left(\alpha_{1} \alpha_{2}\right)^{i} \alpha_{1}, \beta_{1}\right)\right\} & \text { if } n=2 i+1, \\
\emptyset & \text { otherwise }
\end{array}\right. \\
& { }^{-}(0,0)_{n}^{-}= \begin{cases}\left\{\left(\beta_{1}, \alpha_{1}\right)\right\} & \text { if } n=1 \\
\emptyset & \text { otherwise }\end{cases} \\
& (1,0)_{n}^{-}= \begin{cases}\emptyset, & \text { if } n=1, \\
\left\{\left(\beta_{1}\left(\alpha_{2} \alpha_{1}\right)^{i}, \beta_{1}\right)\right\} & \text { if } n=2 i+1, i \geq 1, \\
\left\{\left(\left(\alpha_{2} \alpha_{1}\right)^{i}, \alpha_{2} \beta_{1}\right)\right\} & \text { if } n=2 i,\end{cases} \\
& -(0,1)_{n}= \begin{cases}\left\{\left(\beta_{1}\left(\alpha_{2} \alpha_{1}\right)^{i}, \alpha_{1}\right)\right\} & \text { if } n=2 i+1, i \geq 1, \\
\emptyset & \text { otherwise }\end{cases} \\
& (1,1)_{n}= \begin{cases}\left\{\left(\alpha_{1}, \alpha_{1}\right),\left(\alpha_{2}, \alpha_{2}\right),\left(\beta_{1}, \beta_{1}\right)\right\} & \text { if } n=1, \\
\left\{\left(\left(\alpha_{1} \alpha_{2}\right)^{i} \alpha_{1}, \alpha_{1}\right),\left(\left(\alpha_{2} \alpha_{1}\right)^{i} \alpha_{2}, \alpha_{2}\right)\right\} & \text { if } n=2 i+1, i \geq 1, \\
\emptyset & \text { otherwise, }\end{cases} \\
& { }^{-}(0,0)_{n}^{+}=(1,0)_{n}^{+}={ }^{+}(0,1)_{n}=\emptyset \text {, }
\end{aligned}
$$


and in Example 3.2 we have that

$$
\begin{aligned}
+(0,0)_{n}^{+} & = \begin{cases}\left(A P_{n} / / Q_{0}\right) & \text { if } n \text { is even, } \\
\left\{\left(\alpha_{3} \alpha_{4} \alpha_{5}, \beta_{2}\right)\right\} & \text { if } n=3, \\
\left(A P_{7} / / Q_{0}\right) & \text { if } n=7, \\
\emptyset & \text { otherwise, }\end{cases} \\
(1,0)_{n}^{+} & = \begin{cases}\left\{\left(\alpha_{2} \ldots \alpha_{5}, \alpha_{2} \beta_{2}\right)\right\} & \text { if } n=4, \\
\emptyset, & \text { otherwise, }\end{cases} \\
+(0,1)_{n} & = \begin{cases}\left\{\left(\alpha_{3} \ldots \alpha_{6}, \beta_{2} \alpha_{6}\right)\right\} & \text { if } n=4, \\
\emptyset, & \text { otherwise, }\end{cases} \\
(1,1)_{3} & \left.=\left\{\left(\beta_{1} \beta_{2}\right) \beta_{1}, \beta_{1}\right),\left(\beta_{2} \beta_{1} \beta_{2}, \beta_{2}\right)\right\}, \\
(1,1)_{n} & =\left(A P_{n} / / \mathcal{P}_{1}\right) \quad \text { if } n \text { is odd, } n \neq 3, \\
(1,1)_{n} & =\emptyset \quad \text { if } n \text { is even, } \\
+(0,0)_{n}^{-} & ={ }^{-}(0,0)_{n}^{+}=-(0,0)_{n}^{-}=(1,0)_{n}^{-}=-(0,1)_{n}=\emptyset .
\end{aligned}
$$

We start by describing the behavior of our maps $F_{n+1}^{1}$ restricted to the subsets we have just defined. With this aim in mind, for any $n \geq 1$ we define a map

$$
\phi_{n}:(1,0)_{n}^{+} \rightarrow^{+}(0,1)_{n}
$$

as follows: given $(\rho, \gamma) \in(1,0)_{n}^{+}$we have that $\rho=\alpha \rho^{\prime}, \gamma=\alpha \gamma^{\prime}, \rho^{\prime}$ and $\gamma^{\prime}$ do not share their ending arrows and there exists an arrow $\beta$ such that $\gamma^{\prime} \beta \notin I$. Since $A$ is a string algebra we conclude that $\beta$ is unique and that $\left(\rho^{\prime} \beta, \gamma^{\prime} \beta\right) \in{ }^{+}(0,1)_{n}$. Hence we define $\phi_{n}\left(\alpha \rho^{\prime}, \alpha \gamma^{\prime}\right)=$ $\left(\rho^{\prime} \beta, \gamma^{\prime} \beta\right)$.

The proof of the following lemma is analogous to the proof of [25, Lemma 4.1] concerning triangular string algebras.

Lemma 3.4. For any $n \geq 1$ we have

(a) ${ }^{-}(0,0)_{n}^{-} \sqcup(1,0)_{n}^{-} \sqcup{ }^{-}(0,1)_{n} \sqcup(1,1)_{n} \subset \operatorname{Ker} F_{n+1}^{1}$;

(b) the function $F_{n+1}^{1}$ induces a bijection from ${ }^{-}(0,0)_{n}^{+}$to ${ }^{-}(0,1)_{n+1} \cap\left(A P_{n+1} / / \mathcal{P}_{2}\right)$;

(c) the function $F_{n+1}^{1}$ induces a bijection from ${ }^{+}(0,0)_{n}^{-}$to $(1,0)_{n+1}^{-} \cap\left(A P_{n+1} / / \mathcal{P}_{2}\right)$;

(d) the maps $\phi_{n}:(1,0)_{n}^{+} \rightarrow^{+}(0,1)_{n}$ are bijections and

$$
\begin{aligned}
\left(i d+(-1)^{n} \phi_{n}\right)\left((1,0)_{n}^{+}\right) & \subset \operatorname{Ker} F_{n+1}^{1}, \\
(-1)^{n+1} F_{n+1}^{1}\left((1,0)_{n}^{+}\right) & =(1,1)_{n+1} \cap\left(A P_{n+1} / / \mathcal{P}_{2}\right), \\
F_{n+1}^{1}\left({ }^{+}(0,0)_{n}^{+} \cap\left(A P_{n} / / \mathcal{P}_{1}\right)\right) & =\left(i d+(-1)^{n+1} \phi_{n+1}\right)\left((1,0)_{n+1}^{+}\right) \cap k\left(A P_{n+1} / / \mathcal{P}_{2}\right) .
\end{aligned}
$$


Proof. (a) In order to check that $(\rho, \gamma) \in\left(A P_{n} / / \mathcal{P}_{1}\right)$ belongs to Ker $F_{n+1}^{1}$ we have to prove that if $\beta_{1} \rho$ or $\rho \beta_{2}$ belong to $A P_{n+1}$ then $\beta_{1} \gamma \in I$ and $\gamma \beta_{2} \in I$.

If $(\rho, \gamma) \in{ }^{-}(0,0)_{n}^{-}$then the statement is clear because $Q_{1} \gamma \subset I$ and $\gamma Q_{1} \subset I$.

If $(\rho, \gamma) \in(1,0)_{n}^{-}$, namely $(\rho, \gamma)=(\alpha \hat{\rho}, \alpha \hat{\gamma})$ with $\gamma Q_{1} \subset I$, then $\gamma \beta_{2} \in I$. On the other hand, $\beta_{1} \rho \in A P_{n+1}$ implies that $\beta_{1} \alpha \in I$ and then $\beta_{1} \gamma \in I$.

The proof for ${ }^{-}(0,1)_{n}$ is analogous.

Finally, if $(\rho, \gamma) \in(1,1)_{n}$, this is, $(\rho, \gamma)=(\alpha, \alpha)$ if $n=1$ and if $n \geq 2,(\rho, \gamma)=$ $\left(\alpha_{1} \hat{\rho} \alpha_{2}, \alpha_{1} \hat{\gamma} \alpha_{2}\right)$, again $\beta_{1} \rho, \rho \beta_{2} \in A P_{n+1}$ imply directly $\beta_{1} \alpha, \alpha \beta_{2} \in I$ in the first case, and for $n \geq 2, \beta_{1} \alpha_{1}, \alpha_{2} \beta_{2} \in I$, so $\beta_{1} \gamma \in I$ and $\gamma \beta_{2} \in I$.

(b) Since $A$ is a string algebra if $(\rho, \gamma) \in{ }^{-}(0,0)_{n}^{+}$there exists a unique arrow $\beta$ such that $\gamma \beta \in \mathcal{P}$ and then $\rho \beta \in A P_{n+1}$. It is clear that $(\rho \beta, \gamma \beta) \in \in^{-}(0,1)_{n+1} \cap\left(A P_{n+1} / / \mathcal{P}_{2}\right)$ and $F_{n+1}^{1}((\rho, \gamma))=(-1)^{n+1}(\rho \beta, \gamma \beta)$.

(c) Analogous to the previous one.

(d) By construction it is clear that the map $\phi_{n}$ is a bijection. The first statements follow from the fact that if $\left(\alpha \rho^{\prime}, \alpha \gamma^{\prime}\right) \in(1,0)_{n}^{+}$, then $\phi_{n}\left(\alpha \rho^{\prime}, \alpha \gamma^{\prime}\right)=\left(\rho^{\prime} \beta, \gamma^{\prime} \beta\right),\left(\alpha \rho^{\prime} \beta, \alpha \gamma^{\prime} \beta\right) \in$ $(1,1)_{n+1} \cap\left(A P_{n+1} / / \mathcal{P}_{2}\right)$ and

$$
F_{n+1}^{1}\left(\rho^{\prime} \beta, \gamma^{\prime} \beta\right)=\left(\alpha \rho^{\prime} \beta, \alpha \gamma^{\prime} \beta\right)=(-1)^{n+1} F_{n+1}^{1}\left(\alpha \rho^{\prime}, \alpha \gamma^{\prime}\right) .
$$

Finally if $(\rho, \gamma) \in{ }^{+}(0,0)_{n}^{+} \cap\left(A P_{n} / / \mathcal{P}_{1}\right)$ there exist unique arrows $\alpha, \beta$ such that $\alpha \gamma \beta \in$ $\mathcal{P}$. So $(\alpha \rho, \alpha \gamma) \in(1,0)_{n+1}^{+} \cap\left(A P_{n+1} / / \mathcal{P}_{2}\right), \phi_{n+1}(\alpha \rho, \alpha \gamma)=(\rho \beta, \gamma \beta) \in{ }^{+}(0,1)_{n+1} \cap$ $\left(A P_{n+1} / / \mathcal{P}_{2}\right)$ and

$$
F_{n+1}((\rho, \gamma))=(\alpha \rho, \alpha \gamma)+(-1)^{n+1}(\rho \beta, \gamma \beta)
$$

Proposition 3.5. For any $n \geq 1$ we have that

$$
\begin{aligned}
\operatorname{dim}_{k} \operatorname{Ker} F_{n+1}^{1} & =\left.\right|^{-}(0,0)_{n}^{-}|+|^{-}(0,1)_{n}|+|(1,1)_{n}|+|(1,0)_{n} \mid, \\
\operatorname{dim}_{k} \operatorname{Im} F_{n+1}^{1} & =\left|\left(^{-}(0,1)_{n+1} \sqcup(1,0)_{n+1} \sqcup(1,1)_{n+1}\right) \cap\left(A P_{n+1} / / \mathcal{P}_{2}\right)\right| .
\end{aligned}
$$

Proof. It follows directly since the previous lemma implies that the set

$$
-(0,0)_{n}^{-} \sqcup(1,0)_{n}^{-} \sqcup^{-}(0,1)_{n} \sqcup(1,1)_{n} \sqcup\left(i d+(-1)^{n} \phi_{n}\right)\left((1,0)_{n}^{+}\right)
$$

is a basis of $\operatorname{Ker} F_{n+1}^{1}$, the set

$$
\left({ }^{-}(0,1)_{n+1} \sqcup(1,0)_{n+1}^{-} \sqcup(1,1)_{n+1} \sqcup\left(i d+(-1)^{n+1} \phi_{n+1}\right)\left((1,0)_{n+1}^{+}\right)\right) \cap k\left(A P_{n+1} / / \mathcal{P}_{2}\right)
$$


is a basis of $\operatorname{Im} F_{n+1}^{1}$ and

$$
(1,0)_{n}=(1,0)_{n}^{+} \sqcup(1,0)_{n}^{-} .
$$

The following result will be used in the description of the cup product defined in $\operatorname{HH}^{*}(A)$. For any cocycle $f$ in $\operatorname{Ker} F_{n+1}$ we denote by $\bar{f}$ its equivalence class in $\operatorname{HH}^{n}(A)$.

Proposition 3.6. Let $n>1$ and let

$$
f=\sum_{i=1}^{m} \lambda_{i}\left(\rho_{i}, \gamma_{i}\right) \in \operatorname{Ker} F_{n+1}^{1} \cap k\left(A P_{n} / / \mathcal{P}_{2}\right)
$$

such that $\left(\rho_{i}, \gamma_{i}\right) \notin^{-}(0,0)_{n}^{-}$for all $i$ with $1 \leq i \leq m$. Then $\bar{f}=0$ in $\operatorname{HH}^{n}(A)$.

Proof. From Lemma 3.4 we know that $f$ is a linear combination of elements in

$$
\left((1,0)_{n}^{-} \sqcup^{-}(0,1)_{n} \sqcup(1,1)_{n} \sqcup\left(i d+(-1)^{n} \phi_{n}\right)\left((1,0)_{n}^{+}\right)\right) \cap k\left(A P_{n} / / \mathcal{P}_{2}\right) .
$$

Now,

$$
\begin{aligned}
(1,0)_{n}^{-} \cap\left(A P_{n} / / \mathcal{P}_{2}\right) & =F_{n}^{1}\left({ }^{+}(0,0)_{n-1}^{-}\right) \\
-(0,1)_{n} \cap\left(A P_{n} / / \mathcal{P}_{2}\right) & =F_{n}^{1}\left({ }^{-}(0,0)_{n-1}^{+}\right) \\
(1,1)_{n} \cap\left(A P_{n} / / \mathcal{P}_{2}\right) & =(-1)^{n} F_{n}^{1}\left((1,0)_{n-1}^{+}\right) \\
\left(i d+(-1)^{n} \phi_{n}\right)\left((1,0)_{n}^{+}\right) \cap k\left(A P_{n} / / \mathcal{P}_{2}\right) & =F_{n}^{1}\left(^{+}(0,0)_{n-1}^{+} \cap\left(A P_{n-1} / / \mathcal{P}_{1}\right)\right)
\end{aligned}
$$

and these equalities imply that $f$ belongs to the image of $F_{n}$.

In order to describe the behavior of $F_{n+1}^{0}$ in $k\left(A P_{n} / / Q_{0}\right)$ we need a description of the basis elements of this vector space. Observe that $\left(A P_{n} / / Q_{0}\right) \subset^{+}(0,0)_{n}^{+}$.

Definition 3.7. A pair $\left(\alpha_{1} \cdots \alpha_{n}, e_{s\left(\alpha_{1}\right)}\right) \in\left(A P_{n} / / Q_{0}\right)$ is called incomplete if $\alpha_{n} \alpha_{1} \notin I$, and it is called complete if $\alpha_{n} \alpha_{1} \in I$. We denote by $\mathcal{I}_{n}$ and by $\mathcal{C}_{n}$ the set of incomplete and complete pairs in $\left(A P_{n} / / Q_{0}\right)$, respectively.

The cyclic group $\mathbb{Z}_{n}=<\tau>$ of order $n$ acts on $\mathcal{C}_{n}$, with the action given by

$$
\tau\left(\alpha_{1} \cdots \alpha_{n}, e_{s\left(\alpha_{1}\right)}\right)=\left(\alpha_{n} \alpha_{1} \cdots \alpha_{n-1}, e_{s\left(\alpha_{n}\right)}\right) .
$$

For any $(\rho, e) \in \mathcal{C}_{n}$ we define its order as the first natural number $r$ such that $\tau^{r}(\rho, e)=$ $(\rho, e)$, and consider the norm of this element defined as follows

$$
N(\rho, e)=\sum_{i=0}^{r-1} \tau^{i}(\rho, e) .
$$


Clearly $\tau$ and $N$ induce linear maps $\tau, N: k \mathcal{C}_{n} \rightarrow k \mathcal{C}_{n}$. We consider the following subset of complete pairs

$$
\begin{aligned}
\mathcal{C}_{n}(0)=\left\{\left(\alpha_{1} \cdots \alpha_{n}, e_{s\left(\alpha_{1}\right)}\right) \in \mathcal{C}_{n}:\right. & \\
& \left.\nexists \gamma \in Q_{1} \backslash\left\{\alpha_{n}\right\} \text { and } \nexists \beta \in Q_{1} \backslash\left\{\alpha_{1}\right\} \text { with } \alpha_{n} \beta, \gamma \alpha_{1} \in I\right\} .
\end{aligned}
$$

\section{Definition 3.8.}

(a) A complete pair $\left(\alpha_{1} \cdots \alpha_{n}, e_{s\left(\alpha_{1}\right)}\right)$ is called gentle if $\tau^{m}\left(\alpha_{1} \cdots \alpha_{n}, e_{s\left(\alpha_{1}\right)}\right) \in \mathcal{C}_{n}(0)$ for any $m \in \mathbb{Z}$;

(b) An incomplete pair $\left(\alpha_{1} \cdots \alpha_{n}, e_{s\left(\alpha_{1}\right)}\right)$ is called empty if there is no relation $\beta \gamma \in I$ with $t(\beta)=s\left(\alpha_{1}\right)=s(\gamma)$.

We denote $\mathcal{G}_{n}$ and $\mathcal{E}_{n}$ the set of gentle and empty pairs in $\left(A P_{n} / / Q_{0}\right)$ respectively, and $N \mathcal{G}_{n}, N \mathcal{E}_{n}$ their corresponding complements in $\mathcal{C}_{n}$ and $\mathcal{I}_{n}$ respectively, that is

$$
\left(A P_{n} / / Q_{0}\right)=\mathcal{C}_{n} \sqcup \mathcal{I}_{n}, \quad \mathcal{C}_{n}=\mathcal{G}_{n} \sqcup N \mathcal{G}_{n}, \quad \mathcal{I}_{n}=\mathcal{E}_{n} \sqcup N \mathcal{E}_{n} .
$$

Example 3.9. For the algebra presented in Example 3.1 we have that, for any $n>0$,

$$
\begin{aligned}
N \mathcal{G}_{n} & = \begin{cases}\left.\left.\left\{\left(\alpha_{1} \alpha_{2}\right)^{i}, e_{1}\right),\left(\alpha_{2} \alpha_{1}\right)^{i}, e_{2}\right)\right\} & \text { if } n=2 i, \\
\emptyset & \text { otherwise, }\end{cases} \\
\mathcal{G}_{n} & =\emptyset, \\
N \mathcal{E}_{n} & = \begin{cases}\left.\left\{\beta_{1}\left(\alpha_{2} \alpha_{1}\right)^{i-1} \alpha_{2}, e_{1}\right)\right\} & \text { if } n=2 i, \\
\emptyset & \text { otherwise, }\end{cases} \\
\mathcal{E}_{n} & =\emptyset,
\end{aligned}
$$

and in Example 3.2 we have that

$$
\begin{aligned}
\mathcal{G}_{n} & = \begin{cases}\left(A P_{n} / / Q_{0}\right) & \text { if } n=2 i, \\
\emptyset, & \text { otherwise, }\end{cases} \\
N \mathcal{G}_{n} & =\emptyset, \\
\mathcal{E}_{n} & = \begin{cases}\left\{\left(\alpha_{1} \ldots \alpha_{7}, e_{1}\right)\right\} & \text { if } n=7, \\
\emptyset & \text { otherwise, }\end{cases} \\
N \mathcal{E}_{n} & =\emptyset .
\end{aligned}
$$

In order to describe the map $F_{n+1}^{0}: k\left(A P_{n} / / Q_{0}\right) \rightarrow k\left(A P_{n+1} / / Q_{1}\right)$ it is enough to study its behavior in each direct summand $k \mathcal{G}_{n}, k N \mathcal{G}_{n}, k \mathcal{E}_{n}$ and $k N \mathcal{E}_{n}$. 
Observe that any element in the set $(1,1)_{n+1} \cap\left(A P_{n+1} / / Q_{1}\right)$ is of the form

$$
\left(\alpha_{1} \alpha_{2} \cdots \alpha_{n} \alpha_{1}, \alpha_{1}\right)
$$

where $\left(\alpha_{1} \alpha_{2} \cdots \alpha_{n}, e_{s\left(\alpha_{1}\right)}\right)$ is a complete pair in $\mathcal{C}_{n}$, belonging to either $\mathcal{G}_{n}$ or $N \mathcal{G}_{n}$. Hence we can decompose $(1,1)_{n+1} \cap\left(A P_{n+1} / / Q_{1}\right)$ in the disjoint union

$$
(1,1)_{n+1}^{\mathcal{G}} \sqcup(1,1)_{n+1}^{N \mathcal{G}} .
$$

Lemma 3.10. The sequence

$$
k \mathcal{G}_{n} \stackrel{N}{\longrightarrow} k \mathcal{G}_{n} \stackrel{(1-\tau)}{\longrightarrow} k \mathcal{G}_{n} \stackrel{N}{\longrightarrow} k \mathcal{G}_{n}
$$

is exact.

Proof. It is clear that $N(1-\tau)=(1-\tau) N=0$. Any $x \in k \mathcal{G}_{n}$ can be written as follows

$$
x=\sum_{i} \sum_{j=0}^{m_{i}-1} \lambda_{i j} \tau^{j}\left(\rho_{i}, e_{s_{i}}\right)
$$

with $m_{i}$ the order of $\left(\rho_{i}, e_{s_{i}}\right), \lambda_{i j} \in k$ and $\left(\rho_{k}, e_{s_{k}}\right) \neq \tau^{j}\left(\rho_{i}, e_{s_{i}}\right)$ if $k \neq i$. Now, $N(x)=0$ implies that

$$
0=\sum_{i, j} \lambda_{i j} N \tau^{j}\left(\rho_{i}, e_{s_{i}}\right)=\sum_{i, j} \lambda_{i j} N\left(\rho_{i}, e_{s_{i}}\right)
$$

because $N \tau=N$. This implies that $\sum_{j=0}^{m_{i}-1} \lambda_{i j}=0$, and hence

$$
x=\sum_{i, j} \lambda_{i j} \tau^{j}\left(\rho_{i}, e_{s_{i}}\right)-\sum_{i}\left(\sum_{j=0}^{m_{i}-1} \lambda_{i j}\right)\left(\rho_{i}, e_{s_{i}}\right)=\sum_{i} \sum_{j=1}^{m_{i}-1} \lambda_{i j}\left(\tau^{j}-1\right)\left(\rho_{i}, e_{s_{i}}\right) \in \operatorname{Im}(1-\tau) .
$$

On the other hand, if $(1-\tau) x=0$ then

$$
\sum_{i} \sum_{j=0}^{m_{i}-1} \lambda_{i j} \tau^{j}\left(\rho_{i}, e_{s_{i}}\right)=\sum_{i} \sum_{j=1}^{m_{i}} \lambda_{i(j-1)} \tau^{j}\left(\rho_{i}, e_{s_{i}}\right)
$$

so $\lambda_{i 0}=\lambda_{i j}$ for any $j$, and hence

$$
x=\sum_{i} \sum_{j=0}^{m_{i}-1} \lambda_{i 0} \tau^{j}\left(\rho_{i}, e_{s_{i}}\right)=\sum_{i} \lambda_{i 0} N\left(\rho_{i}, e_{s_{i}}\right) \in \operatorname{Im} N .
$$


Lemma 3.11. If $n \geq 1$ then $F_{n+1}^{0}=G_{n+1}^{1} \oplus G_{n+1}^{2} \oplus G_{n+1}^{3}$ with

$$
\begin{aligned}
G_{n+1}^{1} & : k \mathcal{E}_{n} \rightarrow 0, \\
G_{n+1}^{2} & : k \mathcal{G}_{n} \rightarrow k(1,1)_{n+1}^{\mathcal{G}}, \\
G_{n+1}^{3} & : k\left(N \mathcal{E}_{n} \sqcup N \mathcal{G}_{n}\right) \rightarrow k(1,1)_{n+1}^{N \mathcal{G}} \oplus k\left(\left((1,0)_{n+1} \sqcup(0,1)_{n+1}\right) \cap\left(A P_{n+1} / / Q_{1}\right)\right) .
\end{aligned}
$$

Moreover, $G_{n+1}^{3}$ is injective, $\operatorname{Ker} G_{2 m+1}^{2}=k \mathcal{G}_{2 m} / \operatorname{Im}(1-\tau)$ and

$$
\operatorname{Ker} G_{2 m}^{2}= \begin{cases}0 & \text { if } \operatorname{char} k \neq 2, \\ k \mathcal{G}_{2 m-1} / \operatorname{Im}(1-\tau) & \text { if } \operatorname{char} k=2\end{cases}
$$

Proof. Recall that

$$
F_{n+1}^{0}\left(\alpha_{1} \cdots \alpha_{n}, e_{s\left(\alpha_{1}\right)}\right)=\sum_{\substack{\left\{\beta \in Q_{1}: \beta \alpha_{1} \in I, t(\beta)=s\left(\alpha_{1}\right)\right\}}}\left(\beta \alpha_{1} \cdots \alpha_{n}, \beta\right)+(-1)^{n+1} \sum_{\substack{\left\{\beta \in Q_{1}: \alpha_{n} \beta \in I, s(\beta)=s\left(\alpha_{1}\right)\right\}}}\left(\alpha_{1} \cdots \alpha_{n} \beta, \beta\right)
$$

so it is clear that $F_{n+1}^{0}\left(\mathcal{E}_{n}\right)=0$, and if $\left(\alpha_{1} \cdots \alpha_{n}, e_{s\left(\alpha_{1}\right)}\right) \in \mathcal{G}_{n}$ then

$$
G_{n+1}^{2}\left(\alpha_{1} \cdots \alpha_{n}, e_{s\left(\alpha_{1}\right)}\right)=\left(\alpha_{n} \alpha_{1} \cdots \alpha_{n}, \alpha_{n}\right)+(-1)^{n+1}\left(\alpha_{1} \cdots \alpha_{n} \alpha_{1}, \alpha_{1}\right) \in k(1,1)_{n+1}^{\mathcal{G}} .
$$

Now we shall prove that $\operatorname{Ker} G_{2 m+1}^{2}=k \mathcal{G}_{2 m} / \operatorname{Im}(1-\tau)$. From the commutativity of the diagram

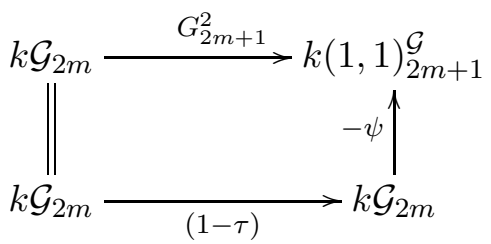

where $\psi\left(\alpha_{1} \cdots \alpha_{2 m}, e_{s\left(\alpha_{1}\right)}\right)=\left(\alpha_{1} \cdots \alpha_{2 m} \alpha_{1}, \alpha_{1}\right)$ is an isomorphism, we get that

$$
\operatorname{Ker} G_{2 m+1}^{2}=\operatorname{Ker}(1-\tau) \text {. }
$$

The equalities

$$
\operatorname{Ker}(1-\tau)=\operatorname{Im} N=k \mathcal{G}_{2 m} / \operatorname{Ker} N=k \mathcal{G}_{2 m} / \operatorname{Im}(1-\tau)
$$

follow from the exactness of the sequence

$$
k \mathcal{G}_{2 m} \stackrel{N}{\longrightarrow} k \mathcal{G}_{2 m} \stackrel{(1-\tau)}{\longrightarrow} k \mathcal{G}_{2 m} \stackrel{N}{\longrightarrow} k \mathcal{G}_{2 m}
$$


proved in Lemma 3.10. If char $k=2$ the same proof works for $\operatorname{Ker} G_{2 m}^{2}$. If char $k \neq 2$, we define $T: k(1,1)_{2 m}^{\mathcal{G}} \rightarrow k \mathcal{G}_{2 m-1}$ as follows:

$$
T\left(\alpha_{1} \cdots \alpha_{2 m-1} \alpha_{1}, \alpha_{1}\right)=\frac{1}{2} \sum_{i=0}^{2 m-2}(-1)^{i} \tau^{i}\left(\alpha_{1} \cdots \alpha_{2 m-1}, e_{s\left(\alpha_{1}\right)}\right)
$$

and we get that $T \circ G_{2 m}^{2}=\mathrm{id}$ and $G_{2 m}^{2} \circ T=\mathrm{id}$, hence $G_{2 m}^{2}$ is bijective.

If $\left(\alpha_{1} \cdots \alpha_{n}, e_{s\left(\alpha_{1}\right)}\right) \in N \mathcal{E}_{n}$ then

$$
\begin{aligned}
& G_{n+1}^{3}\left(\alpha_{1} \cdots \alpha_{n}, e_{s\left(\alpha_{1}\right)}\right) \\
& = \begin{cases}\left(\beta \alpha_{1} \cdots \alpha_{n}, \beta\right) & \in k(1,0)_{n+1}, \\
(-1)^{n+1}\left(\alpha_{1} \cdots \alpha_{n} \gamma, \gamma\right) & \in k(0,1)_{n+1}, \\
\left(\beta \alpha_{1} \cdots \alpha_{n}, \beta\right)+(-1)^{n+1}\left(\alpha_{1} \cdots \alpha_{n} \gamma, \gamma\right) & \in k\left((1,0)_{n+1} \sqcup(0,1)_{n+1}\right)\end{cases}
\end{aligned}
$$

depending on the existence of $\beta$ and $\gamma$ satisfying $\beta \neq \alpha_{n}$ and $\beta \alpha_{1} \in I, \gamma \neq \alpha_{1}$ and $\alpha_{n} \gamma \in I$. Finally, if $\left(\alpha_{1} \cdots \alpha_{n}, e_{s\left(\alpha_{1}\right)}\right) \in N \mathcal{G}_{n}$ then

$$
\begin{aligned}
& G_{n+1}^{3}\left(\alpha_{1} \cdots \alpha_{n}, e_{s\left(\alpha_{1}\right)}\right) \\
& =\left\{\begin{array}{l}
\left(\alpha_{n} \alpha_{1} \cdots \alpha_{n}, \alpha_{n}\right)+(-1)^{n+1}\left(\alpha_{1} \cdots \alpha_{n} \alpha_{1}, \alpha_{1}\right), \\
\left(\alpha_{n} \alpha_{1} \cdots \alpha_{n}, \alpha_{n}\right)+(-1)^{n+1}\left(\alpha_{1} \cdots \alpha_{n} \alpha_{1}, \alpha_{1}\right)+\left(\beta \alpha_{1} \cdots \alpha_{n}, \beta\right), \\
\left(\alpha_{n} \alpha_{1} \cdots \alpha_{n}, \alpha_{n}\right)+(-1)^{n+1}\left(\alpha_{1} \cdots \alpha_{n} \alpha_{1}, \alpha_{1}\right)+(-1)^{n+1}\left(\alpha_{1} \cdots \alpha_{n} \gamma, \gamma\right), \\
\left(\alpha_{n} \alpha_{1} \cdots \alpha_{n}, \alpha_{n}\right)+(-1)^{n+1}\left(\alpha_{1} \cdots \alpha_{n} \alpha_{1}, \alpha_{1}\right)+\left(\beta \alpha_{1} \cdots \alpha_{n}, \beta\right)+(-1)^{n+1}\left(\alpha_{1} \cdots \alpha_{n} \gamma, \gamma\right)
\end{array}\right.
\end{aligned}
$$

depending on the existence of $\beta$ and $\gamma$ in $Q_{1}$ satisfying $\beta \neq \alpha_{n}$ and $\beta \alpha_{1} \in I, \gamma \neq \alpha_{1}$ and $\alpha_{n} \gamma \in I$. Hence

$$
\begin{aligned}
& G_{n+1}^{3}\left(k N \mathcal{E}_{n}\right) \subset k\left(\left((1,0)_{n+1} \sqcup(0,1)_{n+1}\right) \cap\left(A P_{n+1} / / Q_{1}\right)\right), \\
& G_{n+1}^{3}\left(k N \mathcal{G}_{n}\right) \subset k\left(\left((1,1)_{n+1}^{N \mathcal{G}} \sqcup(1,0)_{n+1} \sqcup(0,1)_{n+1}\right) \cap\left(A P_{n+1} / / Q_{1}\right)\right) .
\end{aligned}
$$

Now we define the linear map

$$
T: k\left(\left((1,1)_{n+1}^{N \mathcal{G}} \sqcup(1,0)_{n+1} \sqcup(0,1)_{n+1}\right) \cap\left(A P_{n+1} / / Q_{1}\right)\right) \rightarrow k\left(N \mathcal{E}_{n} \sqcup N \mathcal{G}_{n}\right)
$$


as follows:

$$
\begin{aligned}
T\left(\alpha_{1} \cdots \alpha_{n} \alpha_{1}, \alpha_{1}\right) & =(-1)^{n+1} \sum_{i=0}^{\mu(w)-1}(-1)^{i n} \tau^{i}(w), \\
T\left(\beta \alpha_{1} \cdots \alpha_{n}, \beta\right) & = \begin{cases}w & \text { if } \alpha_{n} \alpha_{1} \notin I, \\
0 & \text { if } \alpha_{n} \alpha_{1} \in I \text { and } \exists \gamma \neq \alpha_{1} \\
(-1)^{n} \sum_{i=0}^{\mu(\tau(w))-1}(-1)^{i n} \tau^{i+1}(w) & \text { such that } \alpha_{n} \gamma \in I,\end{cases} \\
T\left(\alpha_{1} \cdots \alpha_{n} \gamma, \gamma\right) & \text { otherwise, }
\end{aligned}
$$

where $w=\left(\alpha_{1} \cdots \alpha_{n}, e_{s\left(\alpha_{1}\right)}\right)$ and $\mu(w)$ is the first natural number such that $\tau^{\mu(w)-1}(w) \notin$ $\mathcal{C}_{n}(0)$. A direct computation shows that $T \circ G_{n+1}^{3}=\mathrm{id}$, and hence $G_{n+1}^{3}$ is injective.

Proposition 3.12. If $n \geq 1$ then

$$
\begin{aligned}
& \operatorname{dim}_{k} \operatorname{Ker} F_{n+1}^{0}= \begin{cases}\left|\mathcal{E}_{n}\right|+\operatorname{dim}_{k} k \mathcal{G}_{n} / \operatorname{Im}(1-\tau) & \text { if } n \text { is even and char } k \neq 2, \\
\left|\mathcal{E}_{n}\right| & \text { if } n \text { is odd and char } k \neq 2, \\
\left|\mathcal{E}_{n}\right|+\operatorname{dim}_{k} k \mathcal{G}_{n} / \operatorname{Im}(1-\tau) & \text { if } \operatorname{char} k=2,\end{cases} \\
& \operatorname{dim}_{k} \operatorname{Im} F_{n+1}^{0}= \begin{cases}\left|\mathcal{C}_{n}\right|+\left|N \mathcal{E}_{n}\right|-\operatorname{dim}_{k} k \mathcal{G}_{n} / \operatorname{Im}(1-\tau) & \text { if } n \text { is } \text { even } \text { and char } k \neq 2, \\
\left|\mathcal{C}_{n}\right|+\left|N \mathcal{E}_{n}\right| & \text { if } n \text { is odd and char } k \neq 2, \\
\left|\mathcal{C}_{n}\right|+\left|N \mathcal{E}_{n}\right|-\operatorname{dim}_{k} k \mathcal{G}_{n} / \operatorname{Im}(1-\tau) & \text { if char } k=2 .\end{cases}
\end{aligned}
$$

Proof. The formula for the dimension of $\operatorname{Ker} F_{n+1}^{0}$ follows by a direct computation using Lemma 3.11, The equalities

$$
\operatorname{dim}_{k} \operatorname{Im} F_{n+1}^{0}=\left|\left(A P_{n} / / Q_{0}\right)\right|-\operatorname{dim}_{k} \operatorname{Ker} F_{n+1}^{0}
$$

and

$$
\left|\left(A P_{n} / / Q_{0}\right)\right|=\left|\mathcal{G}_{n}\right|+\left|N \mathcal{G}_{n}\right|+\left|\mathcal{E}_{n}\right|+\left|N \mathcal{E}_{n}\right|=\left|\mathcal{C}_{n}\right|+\left|\mathcal{E}_{n}\right|+\left|N \mathcal{E}_{n}\right|
$$

imply the formula for $\operatorname{dim}_{k} \operatorname{Im} F_{n+1}^{0}$. 


\subsection{Dimensions of the Hochschild cohomology groups}

We start this section with the computation of the first Hochschild cohomology groups.

Theorem 3.13. Let $A=k Q / I$ be a quadratic string algebra. Then

$$
\begin{aligned}
& \operatorname{dim}_{k} \operatorname{HH}^{0}(A)=\left.\right|^{-}\left(Q_{0} / / \mathcal{P}_{1}\right)^{-} \mid+1, \\
& \operatorname{dim}_{k} \operatorname{HH}^{1}(A)= \begin{cases}\left|-(0,0)_{1}^{-}\right|+\left|Q_{1}\right|-\left|Q_{0}\right|+1 & \text { if char } k \neq 2, \\
\left.\right|^{-}(0,0)_{1}^{-}|+| Q_{1}|-| Q_{0}|+1+| \mathcal{G}_{1} \mid & \text { if char } k=2 .\end{cases}
\end{aligned}
$$

Proof. Recall that the maps $F_{1}^{0}$ and $F_{1}^{1}$ appearing in the commutative diagram

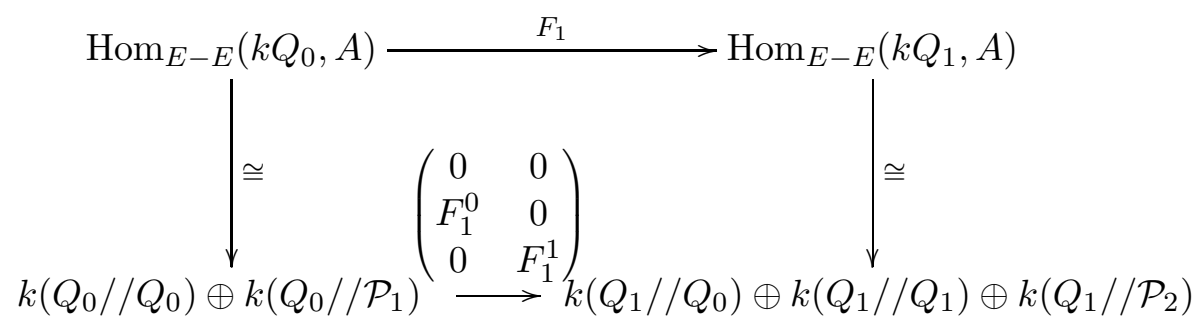

are given by

$$
\begin{aligned}
F_{1}^{0}\left(e_{r}, e_{r}\right) & =\sum_{\left\{\beta \in Q_{1}: t(\beta)=r\right\}}(\beta, \beta)-\sum_{\left\{\beta \in Q_{1}: s(\beta)=r\right\}}(\beta, \beta), \\
F_{1}^{1}\left(e_{r}, \gamma\right) & =\sum_{\left\{\beta \in Q_{1}: t(\beta)=r\right\}}(\beta, \beta \gamma)-\sum_{\left\{\beta \in Q_{1}: s(\beta)=r\right\}}(\beta, \gamma \beta) .
\end{aligned}
$$

Then

$$
F_{1}^{0}\left(\sum_{r \in Q_{0}} \lambda_{r}\left(e_{r}, e_{r}\right)\right)=\sum_{\beta \in Q_{1}}\left(\lambda_{t(\beta)}-\lambda_{s(\beta)}\right)(\beta, \beta)=0
$$

implies that $\lambda_{i}=\lambda_{j}$ whenever there exists an arrow $\beta: i \rightarrow j$. Since $Q$ is connected, we have that $\lambda_{i}=\lambda_{j}$ for any $i, j$. Hence $\operatorname{dim}_{k} \operatorname{Ker} F_{1}^{0}=1$.

On the other hand, $\left(Q_{0} / / \mathcal{P}_{1}\right)=^{-}\left(Q_{0} / / \mathcal{P}_{1}\right)^{-} \sqcup^{-}\left(Q_{0} / / \mathcal{P}_{1}\right)^{+} \sqcup^{+}\left(Q_{0} / / \mathcal{P}_{1}\right)^{-} \sqcup^{+}\left(Q_{0} / / \mathcal{P}_{1}\right)^{+}$ and we have that:

(i) $F_{1}^{1}\left(-\left(Q_{0} / / \mathcal{P}_{1}\right)^{-}\right)=0$;

(ii) $F_{1}^{1}$ induces a bijection from ${ }^{-}\left(Q_{0} / / \mathcal{P}_{1}\right)^{+}$to ${ }^{-}(0,1)_{1}$;

(iii) $F_{1}^{1}$ induces a bijection from ${ }^{+}\left(Q_{0} / / \mathcal{P}_{1}\right)^{-}$to $(1,0)_{1}^{-}$;

(iv) there exists a bijection $\phi_{1}:(1,0)_{1}^{+} \rightarrow^{+}(0,1)_{1}$ given by $\phi_{1}(\alpha, \alpha \gamma)=(\beta, \gamma \beta)$ such that $F_{1}^{1}\left({ }^{+}\left(Q_{0} / / \mathcal{P}_{1}\right)^{+}\right)=\left(i d-\phi_{1}\right)\left((1,0)_{1}^{+}\right)$. 
Hence $^{-}\left(Q_{0} / / \mathcal{P}_{1}\right)^{-}$is a basis for Ker $F_{1}^{1}$ and then

$$
\operatorname{dim}_{k} \operatorname{HH}^{0}(A)=\operatorname{dim}_{k} \operatorname{Ker} F_{1}=\operatorname{dim}_{k} \operatorname{Ker} F_{1}^{0}+\operatorname{dim}_{k} \operatorname{Ker} F_{1}^{1}=1+\left.\right|^{-}\left(Q_{0} / / \mathcal{P}_{1}\right)^{-} \mid .
$$

From these computations we get that

$$
\begin{aligned}
\operatorname{dim}_{k} \operatorname{Im} F_{1}^{0} & =\left|\left(Q_{0} / / Q_{0}\right)\right|-\operatorname{dim}_{k} \operatorname{Ker} F_{1}^{0}=\left|Q_{0}\right|-1, \\
\operatorname{dim}_{k} \operatorname{Im} F_{1}^{1} & =\left|\left(Q_{0} / / \mathcal{P}_{1}\right)\right|-\operatorname{dim}_{k} \operatorname{Ker} F_{1}^{1} \\
& =\left.\right|^{-}(0,1)_{1}|+|(1,0)_{1}^{-}|+|(1,0)_{1}^{+} \mid .
\end{aligned}
$$

From Proposition 3.5 and Proposition 3.12 we have that

$$
\begin{aligned}
\operatorname{dim}_{k} \operatorname{Ker} F_{2} & =\operatorname{dim}_{k} \operatorname{Ker} F_{2}^{0}+\operatorname{dim}_{k} \operatorname{Ker} F_{2}^{1} \\
& =\left|\mathcal{E}_{1}\right|+\left.\right|^{-}(0,0)_{1}^{-}|+|^{-}(0,1)_{1}|+|(1,1)_{1}|+|(1,0)_{1} \mid
\end{aligned}
$$

if $\operatorname{char} k \neq 2$ and

$$
\operatorname{dim}_{k} \operatorname{Ker} F_{2}=\left|\mathcal{E}_{1}\right|+\left.\right|^{-}(0,0)_{1}^{-}|+|^{-}(0,1)_{1}|+|(1,1)_{1}|+|(1,0)_{1} \mid+\operatorname{dim}_{k} k \mathcal{G}_{1} / \operatorname{Im}(1-\tau)
$$

if $\operatorname{char} k=2$. Now $\left|(1,1)_{1}\right|=\left|Q_{1}\right|, \mathcal{E}_{1}=\emptyset$ since $A$ is finite dimensional and

$$
k \mathcal{G}_{1} / \operatorname{Im}(1-\tau)=k \mathcal{G}_{1}
$$

So

$$
\operatorname{dim}_{k} \mathrm{HH}^{1}(A)= \begin{cases}\left|-(0,0)_{1}^{-}\right|+\left|Q_{1}\right|-\left|Q_{0}\right|+1 & \text { if char } k \neq 2, \\ \left.\right|^{-}(0,0)_{1}^{-}|+| Q_{1}|-| Q_{0}|+1+| \mathcal{G}_{1} \mid & \text { if char } k=2\end{cases}
$$

The dimensions that have been computed in Propositions 3.5 and 3.12 lead us to the following theorem.

Theorem 3.14. If $A=k Q / I$ is a quadratic string algebra and $n \geq 2$ then

$$
\begin{aligned}
& \operatorname{dim}_{k} \operatorname{HH}^{n}(A)=\left.\right|^{-}(0,0)_{n}^{-}|+| \mathcal{E}_{n}|-| N \mathcal{E}_{n-1}|+|\left((1,0)_{n} \sqcup^{-}(0,1)_{n}\right) \cap\left(A P_{n} / / Q_{1}\right) \mid \\
& + \begin{cases}\operatorname{dim}_{k} k \mathcal{G}_{n} / \operatorname{Im}(1-\tau) & \text { if } n \text { is even and char } k \neq 2, \\
\operatorname{dim}_{k} k \mathcal{G}_{n-1} / \operatorname{Im}(1-\tau) & \text { if } n \text { is odd and char } k \neq 2, \\
\operatorname{dim}_{k} k \mathcal{G}_{n} / \operatorname{Im}(1-\tau)+\operatorname{dim}_{k} k \mathcal{G}_{n-1} / \operatorname{Im}(1-\tau) & \text { if char } k=2 .\end{cases}
\end{aligned}
$$

Proof. From Propositions 3.12 and 3.5 we get that if $n$ is even and char $k \neq 2$ then

$$
\begin{aligned}
\operatorname{dim}_{k} \mathrm{HH}^{n}(A) & =\operatorname{dim}_{k} \operatorname{Ker} F_{n+1}-\operatorname{dim}_{k} \operatorname{Im} F_{n} \\
& =\left.\right|^{-}(0,0)_{n}^{-}|+|^{-}(0,1)_{n}|+|(1,1)_{n}|+|(1,0)_{n} \mid \\
& -\left|\left(-(0,1)_{n} \sqcup(1,0)_{n} \sqcup(1,1)_{n}\right) \cap\left(A P_{n} / / \mathcal{P}_{2}\right)\right| \\
& +\left|\mathcal{E}_{n}\right|+\operatorname{dim}_{k} k \mathcal{G}_{n} / \operatorname{Im}(1-\tau)-\left|\mathcal{C}_{n-1}\right|-\left|N \mathcal{E}_{n-1}\right|
\end{aligned}
$$


and the desired formula follows since the identification

$$
\left(\alpha_{1} \cdots \alpha_{n-1} \alpha_{1}, \alpha_{1}\right) \leftrightarrow\left(\alpha_{1} \cdots \alpha_{n-1}, e_{s\left(\alpha_{1}\right)}\right)
$$

implies that $\left|(1,1)_{n} \cap\left(A P_{n} / / Q_{1}\right)\right|=\left|\mathcal{C}_{n-1}\right|$. Similarly one can deduce the other formulae.

Definition 3.15. A string algebra $A=k Q / I$ is called a gentle algebra if in addition $(Q, I)$ satisfies :

G1) For an arrow $\alpha$ in $Q$ there exists at most one arrow $\beta$ and at most one arrow $\gamma$ such that $\alpha \beta \in I$ and $\gamma \alpha \in I$;

G2) I is quadratic.

The Hochschild cohomology groups of gentle algebras have already been computed in [20], and these results have been expressed in terms of the derived invariant introduced by AvellaAlaminos and Geiss in [4. As a consequence of our previous theorem, we recover the results in [20].

Corollary 3.16. If $A=k Q / I$ is a gentle algebra, then

$$
\begin{aligned}
& \operatorname{dim}_{k} \mathrm{HH}^{0}(A)=\left.\right|^{-}\left(Q_{0} / / \mathcal{P}_{1}\right)^{-} \mid+1, \\
& \operatorname{dim}_{k} \mathrm{HH}^{1}(A)= \begin{cases}\left|-(0,0)_{1}^{-}\right|+\left|Q_{1}\right|-\left|Q_{0}\right|+1 & \text { if char } k \neq 2, \\
\left.\right|^{-}(0,0)_{1}^{-}|+| Q_{1}|-| Q_{0}|+1+|\left(Q_{1} / / Q_{0}\right) \mid & \text { if } \operatorname{char} k=2,\end{cases} \\
& \operatorname{dim}_{k} \mathrm{HH}^{n}(A)=\left.\right|^{-}(0,0)_{n}^{-}|+| \mathcal{E}_{n} \mid+a \operatorname{dim}_{k} k \mathcal{G}_{n} / \operatorname{Im}(1-\tau)+b \operatorname{dim}_{k} \mathcal{G}_{n-1} / \operatorname{Im}(1-\tau)
\end{aligned}
$$

where

$$
(a, b)= \begin{cases}(1,0) & \text { if } n \geq 2, n \text { even, } \operatorname{char} k \neq 2, \\ (0,1) & \text { if } n \geq 2, n \text { odd }, \text { char } k \neq 2, \\ (1,1) & \text { if } n \geq 2, \text { char } k=2 .\end{cases}
$$

Proof. From Theorems 3.13 and 3.14 it is clear that we only have to prove that

$$
\left|N \mathcal{E}_{n-1}\right|=\left|\left((1,0)_{n} \sqcup^{-}(0,1)_{n}\right) \cap\left(A P_{n} / / Q_{1}\right)\right| .
$$

Since $A$ is gentle, $N \mathcal{G}_{n-1}=\emptyset$, and in this case the injective map

$$
G_{n}^{3}: k\left(N \mathcal{E}_{n-1}\right) \rightarrow k\left(\left((1,0)_{n} \sqcup(0,1)_{n}\right) \cap\left(A P_{n} / / Q_{1}\right)\right)
$$

studied in Lemma 3.11 satisfies

$$
\begin{aligned}
& G_{n}^{3}\left(\alpha_{1} \cdots \alpha_{n-1}, e_{r}\right) \\
& \quad= \begin{cases}\left(\beta \alpha_{1} \cdots \alpha_{n-1}, \beta\right) & \in k\left((1,0)_{n}^{-}\right) \\
(-1)^{n}\left(\alpha_{1} \cdots \alpha_{n-1} \gamma, \gamma\right) & \in k\left(-(0,1)_{n}\right) \\
\left(\beta \alpha_{1} \cdots \alpha_{n-1}, \beta\right)+(-1)^{n}\left(\alpha_{1} \cdots \alpha_{n-1} \gamma, \gamma\right) & \in k\left(\left(i d+(-1)^{n} \phi_{n}\right)(1,0)_{n}^{+}\right)\end{cases}
\end{aligned}
$$


depending on the existence of $\beta$ and $\gamma$. So

$$
\left|N \mathcal{E}_{n-1}\right|=\operatorname{dim}_{k} \operatorname{Im} G_{n}^{3}=\left|\left((1,0)_{n} \sqcup^{-}(0,1)_{n}\right) \cap\left(A P_{n} / / Q_{1}\right)\right| .
$$

Example 3.17. The algebra presented in Example 3.1 is a quadratic string algebra, then by Theorems 3.13 and 3.14 we have that

$$
\operatorname{dim}_{k} \operatorname{HH}^{n}(A)= \begin{cases}2 & \text { if } n=0 \\ 3 & \text { if } n=1, \\ 1 & \text { if } n=2 i+1, i \geq 1 \\ 0 & \text { otherwise. }\end{cases}
$$

The algebra in Example 3.2 is a gentle algebra, then we can compute its cohomology using Corollary 3.16,

$$
\operatorname{dim}_{k} \operatorname{HH}^{n}(A)= \begin{cases}1 & \text { if } n=0 \\ 3 & \text { if } n=1 \\ 2 & \text { if } n=7 \\ 1 & \text { otherwise }\end{cases}
$$

The following results will be used in the description of the Lie bracket defined in $\operatorname{HH}^{*}(A)$.

Proposition 3.18. Let $A$ be a gentle algebra, $n>1$ and let

$$
f=\sum_{i=1}^{m} \lambda_{i}\left(\rho_{i}, \gamma_{i}\right) \in \operatorname{Ker} F_{n+1}^{1}
$$

such that $\left(\rho_{i}, \gamma_{i}\right) \notin^{-}(0,0)_{n}^{-},\left(\rho_{i}, \gamma_{i}\right) \notin(1,1)_{n} \cap\left(A P_{n} / / Q_{1}\right)$ for all $i$ with $1 \leq i \leq m$. Then $\bar{f}=0$ in $\mathrm{HH}^{n}(A)$.

Proof. From Lemma 3.4 we know that $f$ can be written as $f=f_{1}+f_{2}$ where

$$
f_{1} \in \operatorname{Ker} F_{n+1}^{1} \cap k\left(A P_{n} / / Q_{1}\right) \quad \text { and } \quad f_{2} \in \operatorname{Ker} F_{n+1}^{1} \cap k\left(A P_{n} / / \mathcal{P}_{2}\right) .
$$

By Proposition 3.6 we have that $\overline{f_{2}}=0$ in $\operatorname{HH}^{n}(A)$. On the other hand, $f_{1}$ is a linear combination of elements in

$$
\left((1,0)_{n}^{-} \sqcup^{-}(0,1)_{n} \sqcup\left(i d+(-1)^{n} \phi_{n}\right)\left((1,0)_{n}^{+}\right)\right) \cap k\left(A P_{n} / / Q_{1}\right) .
$$

The proof of Corollary 3.16 shows that

$$
\left((1,0)_{n}^{-} \sqcup^{-}(0,1)_{n} \sqcup\left(i d+(-1)^{n} \phi_{n}\right)(1,0)_{n}^{+}\right) \cap k\left(A P_{n} / / Q_{1}\right)=\operatorname{Im} G_{n}^{3} \subset \operatorname{Im} F_{n}^{0} .
$$

Hence $\overline{f_{1}}=0$ in $\operatorname{HH}^{n}(A)$. 
Corollary 3.19. Let $A$ be a gentle algebra, $n>1$ and let

$$
f=\sum_{i=1}^{m} \lambda_{i}\left(\rho_{i}, \gamma_{i}\right) \in \operatorname{Ker} F_{n+1}^{1}
$$

such that $\left(\rho_{i}, \gamma_{i}\right) \notin^{-}(0,0)_{n}^{-}$for all $i$ with $1 \leq i \leq m$. If $\mathcal{G}_{n-1}=\emptyset$ then $\bar{f}=0$ in $\operatorname{HH}^{n}(A)$.

Proof. By assumption $\mathcal{G}_{n-1}=\emptyset$ and, since $A$ is gentle, $N \mathcal{G}_{n-1}=\emptyset$. Then

$$
(1,1)_{n} \cap\left(A P_{n} / / Q_{1}\right)=(1,1)_{n}^{\mathcal{G}} \sqcup(1,1)_{n}^{N \mathcal{G}}=\emptyset
$$

and hence the desired result follows from the previous proposition.

\section{Gerstenhaber algebra}

\subsection{Comparison morphisms}

The cup product and the Lie bracket in the cohomology $\operatorname{HH}^{*}(A)$ are induced by operations defined using the bar resolution, and we have made the computations of $\operatorname{HH}^{n}(A)$ using Bardzell's resolution. In this section we will construct comparison morphisms between the bar resolution and Bardzell's resolution in order to get formulae for the cup product and the Lie bracket.

We start by recalling the definition of these structures at the level of cochains using the bar resolution $\left(A^{\otimes^{n}}, b_{n}\right)_{n \geq 0}$. Given $f \in \operatorname{Hom}_{E-E}\left(A^{\otimes^{n}}, A\right)$ and $g \in \operatorname{Hom}_{E-E}\left(A^{\otimes^{m}}, A\right)$ we have

$$
f \cup g \in \operatorname{Hom}_{E-E}\left(A^{\otimes^{m+n}}, A\right) \quad \text { and } \quad[f, g] \in \operatorname{Hom}_{E-E}\left(A^{\otimes^{m+n-1}}, A\right)
$$

defined by

$$
f \cup g\left(v_{1} \otimes \cdots \otimes v_{n+m}\right)=f\left(v_{1} \otimes \cdots \otimes v_{n}\right) g\left(v_{n+1} \otimes \cdots \otimes v_{n+m}\right)
$$

and

$$
[f, g]=f \circ g-(-1)^{(n-1)(m-1)} g \circ f
$$

where

$$
f \circ g=\sum_{i=1}^{n}(-1)^{(i-1)(m-1)} f \circ_{i} g
$$

and

$$
f \circ_{i} g\left(v_{1} \otimes \ldots \otimes v_{n+m-1}\right)=f\left(v_{1} \otimes \ldots \otimes v_{i-1} \otimes g\left(v_{i} \otimes \ldots v_{i+m-1}\right) \otimes v_{i+m} \otimes \ldots v_{n+m-1}\right) .
$$


These structures are easily carried to Bardzell's resolution using the comparison morphisms

$$
A \otimes k A P_{n} \otimes A \underset{V_{n}}{\stackrel{U_{n}}{\rightleftarrows}} A \otimes A^{\otimes^{n}} \otimes A
$$

given by the $A$-bimodule morphisms

$$
\begin{aligned}
& U_{0}(1 \otimes e \otimes 1)=e \otimes 1, \\
& U_{n}\left(1 \otimes \alpha_{1} \alpha_{2} \ldots \alpha_{n} \otimes 1\right)=1 \otimes \alpha_{1} \otimes \ldots \otimes \alpha_{n} \otimes 1 \quad \text { for } n \geq 1 \text {, } \\
& V_{0}(1 \otimes 1)=1 \otimes 1 \otimes 1=\sum_{i \in Q_{0}} 1 \otimes e_{i} \otimes 1 \\
& V_{1}(1 \otimes \gamma \otimes 1)= \begin{cases}0 & \text { if }|\gamma|=0, \\
\sum_{i=1}^{s} \alpha_{1} \cdots \alpha_{i-1} \otimes \alpha_{i} \otimes \alpha_{i+1} \cdots \alpha_{s} & \text { if } \gamma=\alpha_{1} \cdots \alpha_{s},\end{cases} \\
& V_{n}\left(1 \otimes v_{1} \otimes \cdots \otimes v_{n} \otimes 1\right) \\
& = \begin{cases}v_{1}^{\prime} \otimes \alpha_{1} v_{2} \cdots v_{n-1} \alpha_{n} \otimes v_{n}^{\prime} & \text { if } v_{1}=v_{1}^{\prime} \alpha_{1}, v_{n}=\alpha_{n} v_{n}^{\prime} \\
& \text { and } \alpha_{1} v_{2} \cdots v_{n-1} \alpha_{n} \in A P_{n}, \\
0 & \text { otherwise. }\end{cases}
\end{aligned}
$$

Lemma 4.1. The maps $U=\left(U_{n}\right)_{n \geq 0}$ and $V=(V)_{n \geq 0}$ are morphism of complexes and $V \circ U=\mathrm{id}$.

Using the isomorphisms

$$
\begin{gathered}
\operatorname{Hom}_{A-A}\left(A \otimes A^{\otimes^{n}} \otimes A, A\right) \simeq \operatorname{Hom}_{E-E}\left(A^{\otimes^{n}}, A\right), \\
\operatorname{Hom}_{A-A}\left(A \otimes k A P_{n} \otimes A, A\right) \simeq \operatorname{Hom}_{E-E}\left(k A P_{n}, A\right)
\end{gathered}
$$

we get that the morphisms $U$ and $V$ induce quasi-isomorphisms

$$
\begin{aligned}
& U^{\bullet}=\left(U^{n}: \operatorname{Hom}_{E-E}\left(A^{\otimes^{n}}, A\right) \longrightarrow \operatorname{Hom}_{E-E}\left(k A P_{n}, A\right)\right)_{n \geq 0} \text { and } \\
& V^{\bullet}=\left(V^{n}: \operatorname{Hom}_{E-E}\left(k A P_{n}, A\right) \longrightarrow \operatorname{Hom}_{E-E}\left(A^{\otimes^{n}}, A\right)\right)_{n \geq 0} .
\end{aligned}
$$

With these quasi-isomorphisms, the cup product and the Lie bracket, which we still denote $U$ and [, ], can be defined as follows: given $f \in \operatorname{Hom}_{E-E}\left(k A P_{n}, A\right)$ and $g \in \operatorname{Hom}_{E-E}\left(k A P_{m}, A\right)$,

$$
f \cup g \in \operatorname{Hom}_{E-E}\left(k A P_{n+m}, A\right) \text { and } f \circ_{i} g \in \operatorname{Hom}_{E-E}\left(k A P_{n+m-1}, A\right), 1 \leq i \leq n
$$

are defined by

$$
f \cup g=U^{n+m}\left(V^{n}(f) \cup V^{m}(g)\right) \quad \text { and } \quad f \circ_{i} g=U^{m+n-1}\left(V^{n}(f) \circ_{i} V^{m}(g)\right) .
$$


As usual,

$$
\begin{aligned}
F_{n+m+1}(f \cup g) & =F_{n+1}(f) \cup g+(-1)^{n} f \cup F_{m+1}(g) \\
F_{n+m}([f, g]) & =\left[f, F_{m+1}(g)\right]+(-1)^{m-1}\left[F_{n+1}(f), g\right]
\end{aligned}
$$

so the products $\cup$ and $[-,-]$ defined in the complex $\left(\operatorname{Hom}_{E-E}\left(k A P_{n}, A\right), F_{n}\right)_{n \geq 0}$ induce products at the cohomology level.

\subsection{Formulae for the cup product and the Lie bracket}

The following detailed computations will be useful in order to describe the desired structures:

$$
f \cup g\left(\alpha_{1} \cdots \alpha_{n+m}\right)=f\left(\alpha_{1} \cdots \alpha_{n}\right) g\left(\alpha_{n+1} \cdots \alpha_{n+m}\right)
$$

and, if $g \in \operatorname{Hom}_{E-E}\left(k A P_{m}, A\right)$ is a basis element, that is, $g$ sends a fix basis element in $A P_{m}$ to a basis element in $\mathcal{P}$, and it is zero otherwise, then

$$
f \circ_{1} g\left(\alpha_{1} \ldots \alpha_{n+m-1}\right)=\mu f\left(\beta \alpha_{m+1} \cdots \alpha_{n+m-1}\right)
$$

if $g\left(\alpha_{1} \cdots \alpha_{m}\right)=\mu \beta \in \mathcal{P}_{1}$ and $\beta \alpha_{m+1} \in I$,

$$
f \circ_{n} g\left(\alpha_{1} \ldots \alpha_{n+m-1}\right)=f\left(\alpha_{1} \cdots \alpha_{n-1} \beta\right) \mu
$$

if $g\left(\alpha_{n} \cdots \alpha_{n+m-1}\right)=\beta \mu \in \mathcal{P}_{1}$ and $\alpha_{n-1} \beta \in I$,

$$
f \circ_{i} g\left(\alpha_{1} \cdots \alpha_{n+m-1}\right)=f\left(\alpha_{1} \cdots \alpha_{i-1} \beta \alpha_{i+m} \cdots \alpha_{n+m-1}\right)
$$

if $g\left(\alpha_{i} \cdots \alpha_{i+m-1}\right)=\beta \in Q_{1}$ and $\beta \alpha_{i+m}, \alpha_{i-1} \beta \in I$, and it is zero otherwise.

Now, using the identification $\operatorname{Hom}_{E-E}\left(k A P_{m+n}, A\right) \simeq k\left(A P_{m+n} / / \mathcal{P}\right)$ given by

$$
f_{(\rho, \gamma)} \longleftrightarrow(\rho, \gamma)
$$

we get that, given

$$
(\rho, \gamma)=\left(\alpha_{1} \ldots \alpha_{n}, \gamma\right) \in\left(A P_{n} / / \mathcal{P}\right) \quad \text { and } \quad\left(\rho^{\prime}, \gamma^{\prime}\right)=\left(\beta_{1} \ldots \beta_{m}, \gamma^{\prime}\right) \in\left(A P_{m} / / \mathcal{P}\right)
$$

we have that

- if $\alpha_{n} \beta_{1} \in I$ and $\gamma \gamma^{\prime} \notin I$ then $(\rho, \gamma) \cup\left(\rho^{\prime}, \gamma^{\prime}\right)=\left(\rho \rho^{\prime}, \gamma \gamma^{\prime}\right)$;

- if $\gamma^{\prime} \in Q_{1}, \gamma^{\prime}=\alpha_{i}, \alpha_{i-1} \beta_{1} \in I$ and $\beta_{m} \alpha_{i+1} \in I$ then

$$
(\rho, \gamma) \circ_{i}\left(\rho^{\prime}, \gamma^{\prime}\right)=\left(\alpha_{1} \ldots \alpha_{i-1} \rho^{\prime} \alpha_{i+1} \ldots \alpha_{n}, \gamma\right)
$$

- if $\gamma^{\prime} \in \mathcal{P}_{2}, \gamma^{\prime}=\mu \alpha_{1}$ and $\beta_{m} \alpha_{2} \in I$ then $(\rho, \gamma) \circ_{1}\left(\rho^{\prime}, \gamma^{\prime}\right)=\left(\rho^{\prime} \alpha_{2} \cdots \alpha_{n}, \mu \gamma\right)$;

- if $\gamma^{\prime} \in \mathcal{P}_{2}, \gamma^{\prime}=\alpha_{n} \mu$ and $\alpha_{n-1} \beta_{1} \in I$ then $(\rho, \gamma) \circ_{n}\left(\rho^{\prime}, \gamma^{\prime}\right)=\left(\alpha_{1} \cdots \alpha_{n-1} \rho^{\prime}, \gamma \mu\right)$ and all the other cases are zero. 


\subsection{Vanishing of the cup product and the Lie bracket}

In this section we find conditions on the presentation $(Q, I)$ of the quadratic string algebra that ensures the vanishing of the cup product and the Lie bracket. We start with a remark that will be used throughout this section.

Remark 4.2. Any $\bar{f}=\overline{\sum_{i} \lambda_{i}\left(\rho_{i}, \gamma_{i}\right)}$ in $\operatorname{HH}^{n}(A)$ can be written as $\bar{f}=\overline{f_{1}}+\overline{f_{2}}$ with

$$
f_{1}=\sum_{\left|\gamma_{i}\right|=0} \lambda_{i}\left(\rho_{i}, \gamma_{i}\right) \in \operatorname{Ker} F_{n+1}^{0}, \quad f_{2}=\sum_{\left|\gamma_{i}\right|>0} \lambda_{i}\left(\rho_{i}, \gamma_{i}\right) \in \operatorname{Ker} F_{n+1}^{1}
$$

Moreover from Lemmas 3.4 and 3.11] we have that $f_{1} \in k\left(\mathcal{E}_{n} \sqcup \mathcal{G}_{n}\right)$ and

$$
f_{2} \in k\left(-(0,0)_{n}^{-} \sqcup(1,0)_{n}^{-} \sqcup^{-}(0,1)_{n} \sqcup(1,1)_{n} \sqcup\left(i d+(-1)^{n} \phi_{n}\right)\left((1,0)_{n}^{+}\right)\right) .
$$

Proposition 4.3. Let $A=k Q / I$ be a quadratic string algebra, $n, m>0$. If $\mathcal{G}_{n}=\emptyset=\mathcal{G}_{m}$ then $\mathrm{HH}^{n}(A) \cup \mathrm{HH}^{m}(A)=0$.

Proof. Let $\bar{f}=\overline{f_{1}}+\overline{f_{2}} \in \mathrm{HH}^{n}(A), \bar{g}=\overline{g_{1}}+\overline{g_{2}} \in \mathrm{HH}^{m}(A)$ with $f_{1} \in \operatorname{Ker}_{n+1}^{0}, f_{2} \in$ $\operatorname{Ker} F_{n+1}^{1}, g_{1} \in \operatorname{Ker} F_{m+1}^{0}, g_{2} \in \operatorname{Ker} F_{m+1}^{1}$. We will show that, for any $i, j=1,2$,

$$
\overline{f_{i}} \cup \overline{g_{j}}=\overline{f_{i} \cup g_{j}}=0 .
$$

The assumption $\mathcal{G}_{n}=\emptyset$ and Lemma 3.11 imply that $f_{1}=\sum_{i} \lambda_{i}\left(\rho_{i}, \gamma_{i}\right) \in k\left(\mathcal{E}_{n}\right)$, so for any $\left(\rho^{\prime}, \gamma^{\prime}\right) \in\left(A P_{m} / / \mathcal{P}\right)$ we have that

$$
\left(\rho_{i}, \gamma_{i}\right) \cup\left(\rho^{\prime}, \gamma^{\prime}\right)=0
$$

because $\rho_{i} \rho^{\prime} \notin A P_{n+m}$. Hence $f_{1} \cup g_{j}=0$. Similarly, $f_{i} \cup g_{1}=0$. Finally consider $f_{2} \cup g_{2}$ where

$$
f_{2}=\sum_{i} \mu_{i}\left(\rho_{i}, \gamma_{i}\right) \in \operatorname{Ker} F_{n+1}^{1}, \quad g_{2}=\sum_{j} \mu_{j}^{\prime}\left(\rho_{j}^{\prime}, \gamma_{j}^{\prime}\right) \in \operatorname{Ker} F_{m+1}^{1} .
$$

Recall that

$$
{ }^{-}(0,0)_{n}^{-} \sqcup(1,0)_{n}^{-} \sqcup^{-}(0,1)_{n} \sqcup(1,1)_{n} \sqcup\left(i d+(-1)^{n} \phi_{n}\right)\left((1,0)_{n}^{+}\right)
$$

is a basis of $\operatorname{Ker} F_{n+1}^{1}$ and observe that $f_{2} \cup g_{2} \in \operatorname{Ker} F_{n+m+1}^{1} \cap k\left(A P_{n+m} / / \mathcal{P}_{2}\right)$. If $\left(\rho_{i}, \gamma_{i}\right) \in$ $-(0,0)_{n}^{-}$or $\left(\rho_{j}^{\prime}, \gamma_{j}^{\prime}\right) \in \in^{-}(0,0)_{m}^{-}$then $\gamma_{i} \gamma_{j}^{\prime} \in I$ and hence $\left(\rho_{i} \rho_{j}^{\prime}, \gamma_{j} \gamma_{j}^{\prime}\right)=0$. If $\left(\rho_{i}, \gamma_{i}\right) \in{ }^{-}(0,1)_{n}$ and $\left(\rho_{j}^{\prime}, \gamma_{j}^{\prime}\right) \in(1,0)_{m}^{-}$we have that

$$
\left(\rho_{i}, \gamma_{i}\right)=\left(\hat{\rho}_{i} \alpha_{1}, \hat{\gamma}_{i} \alpha_{1}\right) \quad \text { and } \quad\left(\rho_{j}^{\prime}, \gamma_{j}^{\prime}\right)=\left(\alpha_{2} \hat{\rho}^{\prime}{ }_{j}, \alpha_{2}{\hat{\gamma^{\prime}}}_{j}\right)
$$

with $\alpha_{1}, \alpha_{2} \in Q_{1}$. Then $\left(\rho_{i} \rho_{j}^{\prime}, \gamma_{i} \gamma_{j}^{\prime}\right)=\left(\hat{\rho}_{i} \alpha_{1} \alpha_{2}{\hat{\rho^{\prime}}}_{j}, \hat{\gamma}_{i} \alpha_{1} \alpha_{2}{\hat{\gamma^{\prime}}}_{j}\right)=0$ because $\alpha_{1} \alpha_{2} \in I$ or $\hat{\rho}_{i} \alpha_{1} \alpha_{2}{\hat{\rho^{\prime}}}_{j} \notin A P_{n+m}$. In all the remaining cases $\left(\rho_{i} \rho_{j}^{\prime}, \gamma_{i} \gamma_{j}^{\prime}\right) \notin^{-}(0,0)_{n+m}^{-}$, and the desired result follows from Proposition 3.6 . 
Corollary 4.4. Let $A=k Q / I$ be a quadratic string algebra, char $k \neq 2$. Then $\operatorname{HH}^{n}(A) \cup$ $\mathrm{HH}^{m}(A)=0$ for any $n, m>0$ odd natural numbers.

Proof. The assertion follows from the previous proof since for $n$ odd we have that $\operatorname{Ker} F_{n+1}^{0}=$ $k \mathcal{E}_{n}$ and hence the hypothesis $\mathcal{G}_{n}=\emptyset$ is superfluous in this case.

Now we will describe the Lie bracket for gentle algebras.

Lemma 4.5. If $A=k Q / I$ is a gentle algebra, $n, m>1$ and $g \in{ }^{-}(0,0)_{m}^{-}$. Then $f \circ g=0$ for any $f \in\left(A P_{n} / / \mathcal{P}\right)$.

Proof. Since $f \circ g=\sum_{i=1}^{n}(-1)^{(i-1)(m-1)} f \circ_{i} g$, it suffices to compute $f \circ_{i} g$ for each $i$ with $1 \leq i \leq n$. Let $g=(\rho, \gamma)=\left(\beta_{1} \cdots \beta_{m}, \gamma\right)$, with $\gamma=\mu \gamma^{\prime}=\gamma^{\prime \prime} \nu, \mu, \nu \in Q_{1}, \beta_{1} \neq \mu$ and $\beta_{m} \neq \nu$ and let $f=\left(\alpha_{1} \ldots \alpha_{n}, \delta\right) \in\left(A P_{n} / / \mathcal{P}\right)$. The non vanishing of $f \circ_{i} g$ would imply that

$$
\begin{gathered}
\alpha_{1} \ldots \alpha_{i-1} \beta_{1} \ldots \beta_{m} \alpha_{i+1} \ldots \alpha_{n} \in A P_{n+m-1} \text { and } \alpha_{i}=\mu \\
\text { or } \\
\alpha_{1} \ldots \alpha_{i-1} \beta_{1} \ldots \beta_{m} \alpha_{i+1} \ldots \alpha_{n} \in A P_{n+m-1} \text { and } \alpha_{i}=\nu .
\end{gathered}
$$

In this case

$$
\begin{aligned}
& \alpha_{i-1} \beta_{1} \in I \text { and } \alpha_{i-1} \mu \in I \\
& \text { or } \\
& \beta_{m} \alpha_{i+1} \in I \text { and } \nu \alpha_{i+1} \in I .
\end{aligned}
$$

But $\beta_{1} \neq \mu, \beta_{m} \neq \nu$ and by hypothesis $A$ is gentle. This is a contradiction.

Proposition 4.6. If $A=k Q / I$ is a gentle algebra, $n, m>1$, and $\mathcal{G}_{n-1}=\emptyset=\mathcal{G}_{m-1}$ then $\left[\mathrm{HH}^{n}(A), \mathrm{HH}^{m}(A)\right]=0$.

Proof. Let $\bar{f}=\overline{f_{1}}+\overline{f_{2}} \in \operatorname{HH}^{n}(A), \bar{g}=\overline{g_{1}}+\overline{g_{2}} \in \mathrm{HH}^{m}(A)$, with $f_{1} \in \operatorname{Ker}_{n+1}^{0}, f_{2} \in$ $\operatorname{Ker} F_{n+1}^{1}, g_{1} \in \operatorname{Ker} F_{m+1}^{0}, g_{2} \in \operatorname{Ker} F_{m+1}^{1}$. We will show that $\left[\overline{f_{i}}, \overline{g_{j}}\right]=0$ for any $i, j$. Since $f_{1} \in k\left(A P_{n} / / Q_{0}\right)$ and $g_{1} \in k\left(A P_{m} / / Q_{0}\right)$, it is clear that $f_{i} \circ g_{1}=0=g_{i} \circ f_{1}$ for any $i$. The statement is clear if $\bar{f}_{2}=0=\bar{g}_{2}$. If $\bar{f}_{2} \neq 0$, by Corollary 3.19 we may assume that $f_{2}$ belongs to $k\left(-(0,0)_{n}^{-}\right)$. In this case Lemma 4.5 implies that $g_{i} \circ f_{2}=0$ for any $i$. The case $\bar{g}_{2} \neq 0$ is analogous, and hence we are done.

Corollary 4.7. Let $A=k Q / I$ be a gentle algebra, char $k \neq 2$. Then $\left[\operatorname{HH}^{n}(A), \mathrm{HH}^{m}(A)\right]=0$ for any $n, m \geq 1$ even natural numbers.

Proof. From the previous proof and Proposition 3.18 we deduce that we only have to consider the case $f_{2} \in k(1,1)_{n} \cap k\left(A P_{n} / / Q_{1}\right)$ and $g_{2} \in k(1,1)_{m} \cap k\left(A P_{m} / / Q_{1}\right)$. Since $A$ is gentle we have that $(1,1)_{2 s} \cap\left(A P_{2 s} / / Q_{1}\right)=(1,1)_{2 s}^{\mathcal{G}}$, and the proof of Proposition 3.11 shows that $k(1,1)_{2 s}^{\mathcal{G}}=\operatorname{Im} G_{2 s}^{2} \subset \operatorname{Im} F_{2 s}$, so $\bar{f}_{2}=0$ and $\bar{g}_{2}=0$ in this case. 


\subsection{Non-vanishing of the cup product and the Lie bracket}

In this section we find conditions on the presentation $(Q, I)$ of a quadratic string algebra in order to get non-trivial structures.

Theorem 4.8. Let $A=k Q / I$ be a quadratic string algebra and $\mathcal{G}_{n} \neq \emptyset$ for some $n>0$. Then the cup product defined in $\mathrm{HH}^{*}(A)$ is non-trivial. More precisely,

(i) if $n$ is even and char $k \neq 2, \mathrm{HH}^{s_{1} n}(A) \cup \mathrm{HH}^{s_{2} n}(A) \neq 0$;

(ii) if $n$ is odd and char $k \neq 2, \operatorname{HH}^{2 s_{1} n}(A) \cup \mathrm{HH}^{2 s_{2} n}(A) \neq 0$;

(iii) if $\operatorname{char} k=2, \mathrm{HH}^{s_{1} n}(A) \cup \mathrm{HH}^{s_{2} n}(A) \neq 0$

for any $s_{1}, s_{2} \geq 1$.

Proof. By hypothesis there exists $\omega=\left(\alpha_{1} \cdots \alpha_{n}, e_{s\left(\alpha_{1}\right)}\right) \in \mathcal{G}_{n}$. If this element has order $r$ and char $k=2$ or, char $k \neq 2$ and $n$ is even, we have seen in the proof of Lemma 3.11 that

$$
N(\omega)=N\left(\alpha_{1} \cdots \alpha_{n}, e_{s\left(\alpha_{1}\right)}\right)=\sum_{i=0}^{r-1} \tau^{i}\left(\alpha_{1} \cdots \alpha_{n}, e_{s\left(\alpha_{1}\right)}\right) \in \operatorname{Ker} F_{n+1}^{0} .
$$

Moreover, for any $s \geq 1$, the element $\omega^{s}=\left(\left(\alpha_{1} \cdots \alpha_{n}\right)^{s}, e_{s\left(\alpha_{1}\right)}\right)$ belongs to $\mathcal{G}_{s n}$, has order $r$ and

$$
N\left(\omega^{s}\right)=N\left(\left(\alpha_{1} \cdots \alpha_{n}\right)^{s}, e_{s\left(\alpha_{1}\right)}\right)=\sum_{i=0}^{r-1} \tau^{i}\left(\left(\alpha_{1} \cdots \alpha_{n}\right)^{s}, e_{s\left(\alpha_{1}\right)}\right) \in \operatorname{Ker} F_{s n+1}^{0} .
$$

The element $N\left(\omega^{s}\right)$ is non-zero in $\operatorname{HH}^{s n}(A)$ because $\operatorname{Im} F_{s n} \subseteq k\left(A P_{s n} / / \mathcal{P}_{1}\right)$. For any $s_{1}, s_{2} \geq$ 1 and $0 \leq i, j<r$, we have

$$
\tau^{i}\left(\omega^{s_{1}}\right) \cup \tau^{j}\left(\omega^{s_{2}}\right)=\delta_{i j} \tau^{i}\left(\omega^{s_{1}+s_{2}}\right)
$$

where $\delta_{i j}$ is the Kronecker's delta. Then

$$
N\left(\omega^{s_{1}}\right) \cup N\left(\omega^{s_{2}}\right)=N\left(\omega^{s_{1}+s_{2}}\right)
$$

and hence

$$
\mathrm{HH}^{s_{1} n}(A) \cup \mathrm{HH}^{s_{2} n}(A) \neq(0) .
$$

If $n$ is odd and char $k \neq 2$, we consider the elements $\omega^{2 s}$ and we get that

$$
\mathrm{HH}^{2 s_{1} n}(A) \cup \mathrm{HH}^{2 s_{2} n}(A) \neq(0)
$$

for any $s_{1}, s_{2} \geq 1$. 
Theorem 4.9. Let char $k=0$ and let $A=k Q / I$ be a quadratic string algebra such that $\mathcal{G}_{n} \neq \emptyset$ for some $n>0$. Then the Lie bracket defined in $\operatorname{HH}^{*}(A)$ is non-trivial. More precisely,

(i) if $n$ is even, $\left[\mathrm{HH}^{s_{1} n+1}(A), \mathrm{HH}^{s_{2} n+1}(A)\right] \neq 0$;

(ii) if $n$ is odd, $\left[\mathrm{HH}^{2 s_{1} n+1}(A), \mathrm{HH}^{2 s_{2} n+1}(A)\right] \neq 0$

for any $s_{1}, s_{2} \geq 1, s_{1} \neq s_{2}$.

Proof. By hypothesis there exists $\omega=\left(\alpha_{1} \cdots \alpha_{n}, e_{s\left(\alpha_{1}\right)}\right) \in \mathcal{G}_{n}$ and suppose that it has order $r$. From Lemma 3.4 we have that

$$
\psi(\omega)=\left(\alpha_{1} \ldots \alpha_{n} \alpha_{1}, \alpha_{1}\right) \in \operatorname{Ker} F_{n+2}^{1}
$$

because $\left(\alpha_{1} \ldots \alpha_{n} \alpha_{1}, \alpha_{1}\right) \in(1,1)_{n+1}$. Moreover, for any $s \geq 1$, the element

$$
\omega^{s}=\left(\left(\alpha_{1} \cdots \alpha_{n}\right)^{s}, e_{s\left(\alpha_{1}\right)}\right) \in \mathcal{G}_{s n}
$$

also has order $r$ and

$$
\psi\left(\omega^{s}\right)=\left(\left(\alpha_{1} \cdots \alpha_{n}\right)^{s} \alpha_{1}, \alpha_{1}\right) \in \operatorname{Ker} F_{s n+2}^{1} .
$$

If $n$ is even, the element $\psi\left(\omega^{s}\right)$ is non zero in $\mathrm{HH}^{s n+1}(A)$ because we have seen in the proof of Lemma 3.11 that $\psi\left(\omega^{s}\right) \in \operatorname{Im} F_{s n+1}$ if and only if $w^{s} \in \operatorname{Im}(1-\tau)$. But $\operatorname{Im}(1-\tau)=\operatorname{Ker} N$ and $w^{s} \notin \operatorname{Ker} N$. For any $s_{1}, s_{2} \geq 1$, we have that

$$
\psi\left(\omega^{s_{1}}\right) \circ_{i} \psi\left(\omega^{s_{2}}\right)= \begin{cases}\psi\left(\omega^{s_{1}+s_{2}}\right), & \text { if } i=l r+1, \text { for } l=0, \cdots, s_{1} \frac{n}{r} \\ 0, & \text { otherwise. }\end{cases}
$$

Then

$$
\begin{aligned}
\psi\left(\omega^{s_{1}}\right) \circ \psi\left(\omega^{s_{2}}\right) & =\sum_{i=1}^{s_{1} n+1}(-1)^{(i-1)\left(s_{2} n\right)} \psi\left(\omega^{s_{1}}\right) \circ_{i} \psi\left(\omega^{s_{2}}\right)=\sum_{l=0}^{s_{1} \frac{n}{r}}(-1)^{(l r)\left(s_{2} n\right)} \psi\left(\omega^{s_{1}+s_{2}}\right) \\
& =\left(s_{1} \frac{n}{r}+1\right) \psi\left(\omega^{s_{1}+s_{2}}\right)
\end{aligned}
$$

and hence

$$
\begin{aligned}
{\left[\psi\left(\omega^{s_{1}}\right), \psi\left(\omega^{s_{2}}\right)\right] } & =\psi\left(\omega^{s_{1}}\right) \circ \psi\left(\omega^{s_{2}}\right)-(-1)^{\left(s_{1} n\right)\left(s_{2} n\right)} \psi\left(\omega^{s_{2}}\right) \circ \psi\left(\omega^{s_{1}}\right) \\
& =\left(s_{1} \frac{n}{r}+1\right) \psi\left(\omega^{s_{1}+s_{2}}\right)-\left(s_{2} \frac{n}{r}+1\right) \psi\left(\omega^{s_{1}+s_{2}}\right) \\
& =\frac{n}{r}\left(s_{1}-s_{2}\right) \psi\left(\omega^{s_{1}+s_{2}}\right) .
\end{aligned}
$$


So,

$$
\left[\operatorname{HH}^{n s_{1}+1}(A), \mathrm{HH}^{n s_{2}+1}(A)\right] \neq 0 \text { if } s_{1} \neq s_{2} .
$$

If $n$ is odd, we consider the element

$$
\psi\left(\omega^{2 s}\right)=\left(\left(\alpha_{1} \ldots \alpha_{n}\right)^{2 s} \alpha_{1}, \alpha_{1}\right) \in \operatorname{Ker} F_{2 s n+2}
$$

and we get that

$$
\left[\mathrm{HH}^{2 n s_{1}+1}(A), \mathrm{HH}^{2 n s_{2}+1}(A)\right] \neq 0 \text {, if } s_{1} \neq s_{2} \text {. }
$$

Remark 4.10. The previous theorem also holds in any characteristic if we add some hypothesis on the number $\frac{n}{r}$.

Example 4.11. For the algebra in Example 3.2 we have that $\mathcal{G}_{n} \neq \emptyset$ hence our Theorems 4.9 and 4.8 say that its Gerstenhaber algebra structure is non-trivial. More precisely, if we consider the following sets of generators:

- $\left\{u_{1}=\left(\beta_{1}, \beta_{1}\right), v_{1}=\left(\alpha_{1}, \alpha_{1}\right), w_{1}=\left(\alpha_{7}, \alpha_{7}\right)\right\}$ for $\operatorname{HH}^{1}(A)$,

- $\left\{u_{7}=\left(\left(\beta_{1} \beta_{2}\right)^{3} \beta_{1}, \beta_{1}\right), v_{7}=\left(\alpha_{1} \ldots \alpha_{7}, e_{1}\right)\right\}$ for $\operatorname{HH}^{7}(A)$,

- $\left\{\left(u_{2 n+1}=\left(\left(\beta_{1} \beta_{2}\right)^{n} \beta_{1}, \beta_{1}\right)\right\}\right.$ for $\mathrm{HH}^{2 n+1}(A)$ when $n>0, n \neq 3$, and

- $\left\{u_{2 n}=N\left(\left(\left(\beta_{1} \beta_{2}\right)^{n}, e_{6}\right)\right)\right\}$ for $\operatorname{HH}^{2 n}(A)$ when $n \geq 1$,

then

$$
\begin{aligned}
u_{2 i} \cup u_{2 j} & =u_{2(i+j)} \\
u_{2 i} \cup u_{2 j+1} & =u_{2 j+1} \cup u_{2 i}=u_{2(i+j)+1} \\
{\left[u_{2 i+1}, u_{2 j+1}\right] } & =(i-j) u_{2(i+j)+1} \\
{\left[u_{2 i}, u_{2 j+1}\right] } & =-\left[u_{2 j+1}, u_{2 i}\right]=i u_{2(i+j)} \\
{\left[v_{7}, v_{1}\right] } & =-\left[v_{1}, v_{7}\right]=v_{7} \\
{\left[v_{7}, w_{1}\right] } & =-\left[w_{1}, v_{7}\right]=v_{7}
\end{aligned}
$$

and all the other cases are zero. 
Example 4.12. Let char $k \neq 2$ and let $A=k Q / I$ be the radical square zero algebra whose quiver $Q$ consists of an oriented cycle of length $m>1$

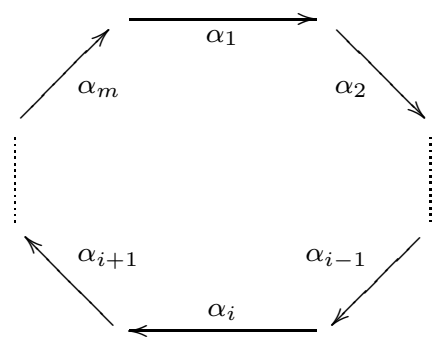

A straightforward computation shows that

$$
\mathrm{HH}^{q m}(A)=k, \quad \mathrm{HH}^{q m+1}(A)=k
$$

when $q m$ is even and it is zero in all the other cases. More precisely,

$$
u_{q m}=N\left(\left(\alpha_{1}, \ldots, \alpha_{m}\right)^{q}, e_{s\left(\alpha_{1}\right)}\right) \quad \text { and } \quad u_{q m+1}=\left(\left(\alpha_{1} \ldots, \alpha_{m}\right)^{q} \alpha_{1}, \alpha_{1}\right)
$$

are generators of $\mathrm{HH}^{q m}(A)$ and $\mathrm{HH}^{q m+1}(A)$ respectively. From Theorems 4.8 and 4.9 we know that its Gerstenhaber algebra structure is non-trivial and the bracket on the generators is given by

$$
\begin{aligned}
{\left[u_{q_{1} m+1}, u_{q_{2 m+1}}\right] } & =\left(q_{1}-q_{2}\right) u_{\left(q_{1}+q_{2}\right) m+1} \\
{\left[u_{q_{1} m}, u_{q_{2 m+1}}\right] } & =-\left[u_{q_{2 m+1}}, u_{q_{1} m}\right]=q_{1} u_{\left(q_{1}+q_{2}\right) m}
\end{aligned}
$$

If we only consider odd degrees, that is,

$$
\mathrm{HH}^{o d d}(A)=\bigoplus_{\substack{q \geq 0, \mathrm{e} \\ q m \text { even }}} \mathrm{HH}^{q m+1}(A),
$$

then the Gerstenhaber bracket endows $\mathrm{HH}^{\text {odd }}(A)$ with a Lie algebra structure such that it is isomorphic to the infinite dimensional Witt algebra. In this way we recover the results in [29, Proposition 4.3.4].

\section{References}

[1] Assem, Ibrahim; Happel, Dieter. Generalized tilted algebras of type $A_{n}$, Comm. Algebra, 9 (1981), no. 20, 2101-2125. 
[2] Assem, Ibrahim; Simson, Daniel; Skowroński, Andrzej. Elements of the representation theory of associative algebras, Vol. 1. Techniques of representation theory, London Mathematical Society Student Texts 65, Cambridge University Press, Cambridge, 2006. $\mathrm{x}+458 \mathrm{pp}$.

[3] Assem, Ibrahim; Skowroński, Andrzej. Iterated tilted algebras of type $\tilde{\mathbf{A}}_{n}$, Math. Z. 195 (1987), no. 2, 269-290.

[4] Avella-Alaminos, Diana; Geiss, Christof. Combinatorial derived invariants for gentle algebras, J. Pure Appl. Algebra 212 (2008), no. 1, 228-243.

[5] Bardzell, Michael J. The alternating syzygy behavior of monomial algebras, J. Algebra 188 (1997), no.1, 69-89.

[6] Bardzell, M. J.; Locateli, Ana Claudia; Marcos, Eduardo N. On the Hochschild cohomology of truncated cycle algebras, Comm. Algebra 28 (2000), no. 3, 1615-1639.

[7] Bustamante, Juan Carlos. The cohomology structure of string algebras, J. Pure Appl. Algebra 204 (2006), no. 3, 616-626.

[8] Butler, M. C. R.; Ringel, Claus Michael. Auslander-Reiten sequences with few middle terms and applications to string algebras, Comm. Algebra 15 (1987), no. 1-2, 145-179.

[9] Cibils, Claude. On the Hochschild cohomology of finite-dimensional algebras, Comm. Algebra 16 (1988), no. 3, 645-649.

[10] Cibils, Claude. Hochschild cohomology algebra of radical square zero algebras, Algebras and modules, II, Geiranger. CMS Conf. Proc. 24, Amer. Math. Soc., Providence, RI (1998), 93-101.

[11] Cibils, Claude; Redondo, María Julia; Saorín, Manuel. The first cohomology group of the trivial extension of a monomial algebra, J. Algebra Appl. 3 (2004), no. 2, 143-159.

[12] Crawley-Boevey, William. Tameness of biserial algebras, Arch. Math. (Basel) 65 (1995), no. 5, 399-407.

[13] Erdmann, K. Algebras and dihedral defect groups, Proc. London Math. Soc. (3) 54 (1987), no. $1,88-114$.

[14] Fuller, Kent R. Biserial rings, Ring theory, Proc. Conf., Univ. Waterloo, Waterloo, 1978. Lecture Notes in Math. 734 (1979), Springer, Berlin, 64-90.

[15] Gel'fand, I. M.; Ponomarev, V. A. Indecomposable representations of the Lorentz group, Uspehi Mat. Nauk 23 (1968), no. 2 (140), 3-60. 
[16] Gerstenhaber, Murray. The cohomology structure of an associative ring, Ann. of Math. (2) 78 (1963), 267-288.

[17] Happel, Dieter. Hochschild cohomology of finite-dimensional algebras, Séminaire d'Algèbre Paul Dubreil et Marie-Paul Malliavin, 39ème Année, Paris, 1987/1988. Lecture Notes in Math. 1404 (1989), Springer, Berlin, 108-126.

[18] Janusz, G. J. Indecomposable modules for finite groups, Ann. of Math. (2) 89 (1969), 209-241.

[19] Kawada, Yutaka. On Köthe's problem concerning algebras for which every indecomposable module is cyclic. I, Sci. Rep. Tokyo Kyoiku Daigaku Sect. A 7 (1962), 154-230.

[20] Ladkani, S. Hochschild cohomology of gentle algebras, available at arXiv:1208.2230v1 [math.RT].

[21] Le, Jue; Zhou, Guodong. On the Hochschild cohomology ring of tensor products of algebras, J. Pure Appl. Algebra 218 (2014), no. 8, 1463-1477.

[22] Liu, Yuming; Zhou, Guodong. The Batalin-Vilkovisky structure over the Hochschild cohomology ring of a group algebra, J. Noncommut. Geom. 10 (2016), no. 3, 811-858.

[23] Nakayama, Tadasi. On Frobeniusean algebras. I, Ann. of Math. (2) 40 (1939), 611-633.

[24] Redondo, María Julia. Hochschild cohomology via incidence algebras, J. Lond. Math. Soc. (2) 77 (2008), no. 2, 465-480.

[25] Redondo, María Julia; Román, Lucrecia. Hochschild cohomology of triangular string algebras and its ring structure, J. Pure Appl. Algebra 218 (2014), no. 5, 925-936.

[26] Ringel, Claus Michael. The indecomposable representations of the dihedral 2-groups, Math. Ann. 214 (1975), 19-34.

[27] Ringel, Claus Michael. Kawada's theorem, Abelian group theory, Oberwolfach, 1981. Lecture Notes in Math. 874 (1981), Springer, Berlin-New York, 431-447.

[28] Ringel, Claus Michael. The minimal representation-infinite algebras which are special biserial, Representations of algebras and related topics, EMS Ser. Congr. Rep., Eur. Math. Soc., Zürich, 2011, 501-560.

[29] Sánchez-Flores, Selene. La structure de Lie de la cohomologie de Hochschild d'algèbres monomiales, Université Montpellier II - Sciences et Techniques du Languedoc, 2009, HAL ID: tel-00464064. 
[30] Sánchez-Flores, Selene. The Lie module structure on the Hochschild cohomology groups of monomial algebras with radical square zero, J. Algebra 320 (2008), no. 12, 4249-4269.

[31] Schroll, Sibylle. Trivial extensions of gentle algebras and Brauer graph algebras, J. Algebra 444 (2015), 183-200.

[32] Shepler, Anne V.; Witherspoon, Sarah. Group actions on algebras and the graded Lie structure of Hochschild cohomology, J. Algebra 351 (2012), 350-381.

[33] Sköldberg, Emil. A contracting homotopy for Bardzell's resolution, Math. Proc. R. Ir. Acad. 108 (2008), no. 2, 111-117.

[34] Skowroński, Andrzej; Waschbüsch, Josef. Representation-finite biserial algebras, J. Reine Angew. Math. 345 (1983), 172-181.

[35] Snashall, Nicole; Taillefer, Rachel. The Hochschild cohomology ring of a class of special biserial algebras, J. Algebra Appl. 9 (2010), no. 1, 73-122.

[36] Strametz, Claudia. The Lie algebra structure on the first Hochschild cohomology group of a monomial algebra, J. Algebra Appl. 5 (2006), no. 3, 245-270.

[37] Suárez-Álvarez, Mariano. Applications of the change-of-rings spectral sequence to the computation of Hochschild cohomology, available at arXiv:0707.3210 [math.KT].

[38] Wald, Burkhard; Waschbüsch, Josef. Tame biserial algebras, J. Algebra 95 (1985), no. $2,480-500$.

[39] Witherspoon, Sarah; Zhou, Guodong. Gerstenhaber brackets on Hochschild cohomology of quantum symmetric algebras and their group extensions, Pacific J. Math. 283 (2016), no. $1,223-255$. 